\title{
Vortex-induced buckling of a viscous drop impacting a pool
}

\author{
Er Qiang Li, ${ }^{1,2}$ Daniel Beilharz, ${ }^{3}$ and Sigurdur T. Thoroddsen ${ }^{1}$ \\ ${ }^{1}$ Division of Physical Sciences and Engineering and Clean Combustion Research Center, King Abdullah \\ University of Science and Technology (KAUST), Thuwal 23955-6900, Saudi Arabia \\ ${ }^{2}$ Department of Modern Mechanics, University of Science and Technology of China, Hefei 230027, China \\ ${ }^{3}$ LadHyX, École polytechnique, 91128 Palaiseau and PMMH, ESPCI Paris, 75005 Paris, France
}

(Received 22 November 2016; published 19 July 2017)

\begin{abstract}
We study the intricate buckling patterns which can form when a viscous drop impacts a much lower viscosity miscible pool. The drop enters the pool by its impact inertia, flattens, and sinks by its own weight while stretching into a hemispheric bowl. Upward motion along the outer bottom surface of this bowl produces a vortical boundary layer which separates along its top and rolls up into a vortex ring. The vorticity is therefore produced in a fundamentally different way than for a drop impacting a pool of the same liquid. The vortex ring subsequently advects into the bowl, thereby stretching the drop liquid into ever thinner sheets, reaching the micron level. The rotating motion around the vortex pulls in folds to form multiple windings of double-walled toroidal viscous sheets. The axisymmetric velocity field thereby stretches the drop liquid into progressively finer sheets, which are susceptible to both axial and azimuthal compression-induced buckling. The azimuthal buckling of the sheets tends to occur on the inner side of the vortex ring, while their folds can be stretched and straightened on the outside edge. We characterize the total stretching from high-speed video imaging and use particle image velocimetry to track the formation and evolution of the vortex ring. The total interfacial area between the drop and the pool liquid can grow over 40 -fold during the first $50 \mathrm{~ms}$ after impact. Increasing pool viscosity shows entrapment of a large bubble on top of the drop, while lowering the drop viscosity produces intricate buckled shapes, appearing at the earliest stage and being promoted by the crater motions. We also present an image collage of the most intriguing and convoluted structures observed. Finally, a simple point-vortex model reproduces some features from the experiments and shows variable stretching along the wrapping sheets.
\end{abstract}

DOI: 10.1103/PhysRevFluids.2.073602

\section{INTRODUCTION}

The study of drops impacting into pools of different liquids and the various resulting shapes dates back to 1885 with the work of Thomson and Newall [1]. Among the various intriguing phenomena they observed is the well-known cascade of falling plumes, which often occur during the descent of heavy drops [2-4]. Similar Rayleigh-Taylor-type instabilities also arise for heavy suspension droplets $[2,5,6]$. Heavy particles inside the drop can lead to formation of a Hill-type vortex, with a toroidal structure which can become destabilized to form localized plumes.

The buoyancy-driven stirring and mixing of different viscosity liquids is a fundamental process in the chemical industry as well as in geophysics. Numerous studies have focused on these dynamics for buoyancy-driven blobs of both miscible or immiscible liquids. Landeau et al. [7] have reviewed such experiments of relevance to astrophysical impacts of proto-planetesimals during planet formation and how heavy intrusions affect the mixing of the constitutive elements. The convoluted shapes arising during the breakup can enhance the mixing [8,9]. The fragmentation of drops impacting immiscible pools has been characterized by Lhuissier et al. [10]. Water drops impacting immiscible silicone oils result in convoluted crater evolution [11]. These studies have not identified buckling (the focus of this study) as a significant factor in the stirring of the drop within the pool, whereas our results suggest this may be important. In Fig. 1, we show typical examples of this buckling, which can occur both in the axial and azimuthal directions. 

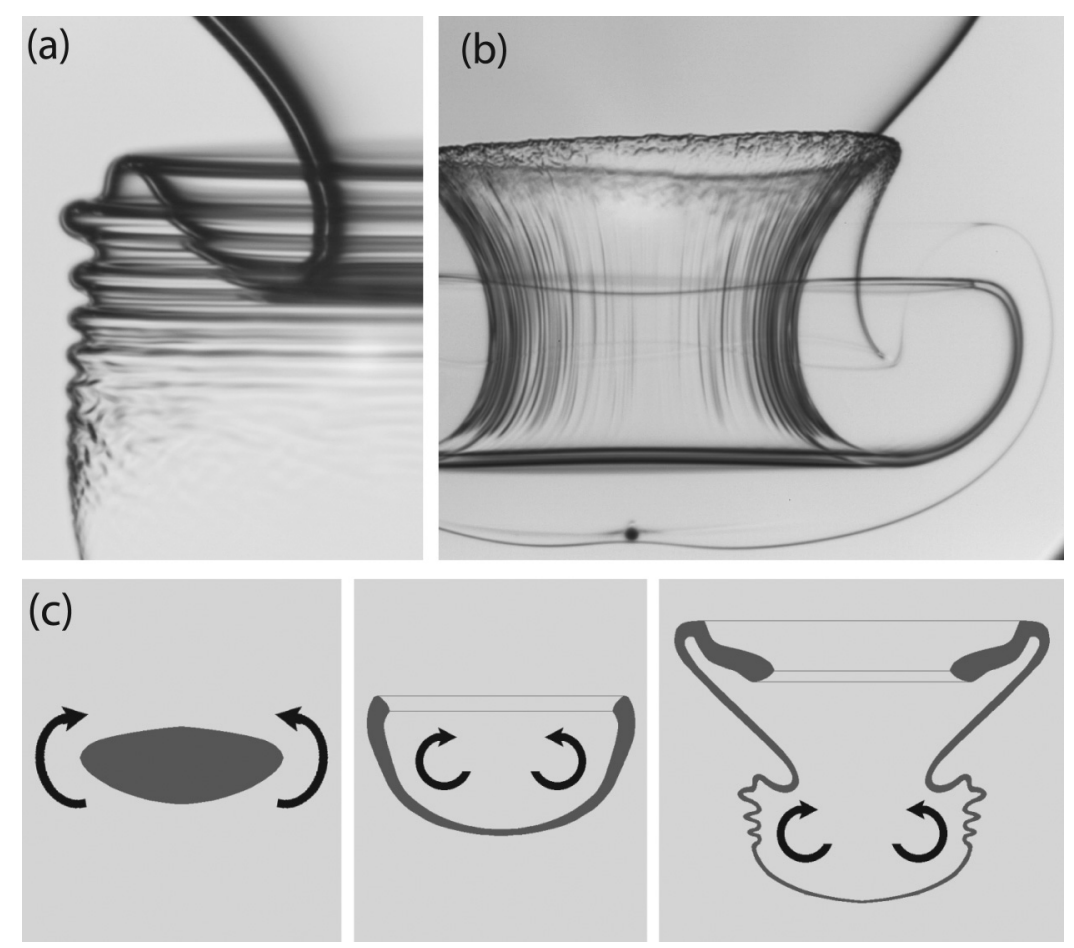

FIG. 1. Examples of the buckling of viscous sheets formed by the impact of a viscous drop onto a lowerviscosity pool. (a) Axisymmetric buckling and (b) azimuthal buckling. (c) Schematic showing that a vortex ring plays a pivotal role in stretching and wrapping these viscous sheets. This ring is produced from the vortical boundary layer in the flow up along the bottom of the drop which separates at the sharp curvature at the sides, then enters the bowl.

Viscous jets and sheets can buckle when subjected to compressive stress, just like the rods and plates in solid mechanics. This is perhaps most familiar for a jet of honey coiling onto a pancake [12-14]. There is even supercoiling when the primary coil becomes long enough [15]. Theories have been formulated in great detail [16,17]. Buckles can appear during the rupture of bubbles at the surface of viscous pools $[18,19]$ or thin liquid films subjected to shear in a Taylor-Couette-type device [20-22]. Buckles also appear during indentation of floating elastic films [23] or at the edges of partially wetting lenses [24].

Cubaud and Mason [25] have studied the folding of a viscous thread flowing within a microchannel and surrounded by a less viscous miscible liquid. The thread is produced in a flow-focusing device. Here, the compression is accomplished by slowing down the viscous thread as it moves from a square channel into a diverging flat channel. The folding frequency is related to the characteristic shear rate, formed by the thread translational velocity and the channel half-width. The folding greatly enhances mixing between the two liquids.

Merrer et al. [26] studied the buckling of a slender filament of viscous liquid surrounded by air, when the air axially compresses the filament at constant velocity. For slender filaments and large enough capillary numbers, they found the buckling was analogous to Euler elastic buckling, whereas at lower compression rates the surface tension can suppress the buckling. Experiments with viscous silicon oils and honey showed similar results.

Liquid buckling is not limited to large viscosities but can appear for water if the compression rate is rapid enough, as occurs during the collapse of cylindrical crowns in sphere impacts [27] and laser ablation [28]. 
Walker et al. [29] have recently studied high-viscosity drops impacting on miscible lowerviscosity pools as we do. Their comprehensive study categorizes the various phenomena, mentioning some of the overall shapes we study, naming some of the intriguing features, for example, lens, jellyfish, and tiers. Their article does not mention any fine buckling, which is the main focus of our study. They also do not measure the velocity fields. Our contribution is the study of the fine details of these intriguing phenomena and identifying the underlying vortical structures.

Most recently, the shape evolution during related impact configurations has been suggested for manufacturing shaped particles through what is called vortex ring freezing [30]. This is accomplished through gelation of the toroidal impact structures of nanoclay droplets.

Vortex rings are most commonly demonstrated as smoke rings but are easily formed by impulsively forcing fluids through any circular opening [31]. Vortex rings are also formed when a drop impacts at low velocity onto a pool of the same liquid [1,32-35]. While smoke rings derive their vorticity from the viscous boundary layer separating from the solid surface, for drop-induced vortex rings the vorticity production is more subtle [36]. Here it arises from the motion of the liquid along a curved free surface, as discussed by Thoraval et al. [37]. Recent experiments have used x-ray imaging to see the details of these vortices [38,39]. Lee et al. [39] use slightly different liquids in drop and pool to clearly show the interface between the liquids using phase-contrast $\mathrm{x}$-ray imaging. In the present study, we show that the vorticity is generated at the boundary between the highly viscous drop and the pool, which differs fundamentally from the homogeneous case above.

Herein, we present a well-controlled experiment where a viscous drop enters a less viscous miscible pool to study the subsequent stirring and stretching of the drop liquid into fine convoluted viscous sheets. We identify certain parameters where the convoluted axisymmetric viscous shapes are susceptible to localized buckling. We use an experimental configuration similar to our recent work on the entrapment of extended air films in Ref. [40], but at higher impact velocities where the air films rupture during early contact, to entrap only a small air disk under the center of the drop [41].

The structure of the paper is the following: First, in Sec. II we describe the high-speed experimental setup and the particle image velocimetry (PIV) technique we use. In Secs. III A-C, we first show overall deformation and buckling results for drops of $500 \mathrm{cSt}$ viscosity impacting pools of much lower viscosity. In Sec. III D, we present direct measurements of the total area of the drop as it is stretch into fine viscous sheets. We then present a simple point-vortex model in Sec. IIIE, which displays some of the observed kinematics. This is followed in Sec. III F by PIV measurements of the underlying vortex ring generated during the impact. In Sec. IV, we investigate how increasing the pool viscosity entraps regular bubbles on top of the drop and how reducing the drop viscosity to $100 \mathrm{cSt}$ produces even more complicated shapes. Finally, we summarize and conclude our findings in Sec. V.

\section{EXPERIMENTAL SETUP}

\section{A. Liquid properties and parameter space}

The drops are pinched off by gravity from a metallic nozzle, then free fall onto a lower viscosity liquid pool. Figure 2 shows a schematic of the setup, which mirrors that used by Beilharz et al. [40]. The pool liquid is contained in a 50-mm-deep glass container, with a $40 \times 50 \mathrm{~mm}$ cross section. The container width is more than 16 times the drop diameter. The large viscosity of the drop liquid rapidly dampens out any pinch-off oscillations, making the drops near spherical at impact, as is shown in the inset in Fig. 2. In most of the experiments, we use a 500-cSt silicone-oil drop, pinched-off from a 1.6-mm-outer-diameter metallic nozzle. The silicone oil wets the nozzle and the pinch-off produces a drop diameter of $D \simeq 2.42 \mathrm{~mm}$. Some experiments were done with a lower viscosity drop of $100 \mathrm{cSt}$, while keeping the same $D$.

The droplet shape evolution within the pool is imaged using a long-distance microscope (Leica Z16 APO) at magnifications up to $14.7 \times$. This gives a pixel resolution down to $1.94 \mu \mathrm{m} / \mathrm{px}$. High-speed videos of the intricate motions are acquired with a Phantom V1610-CMOS video 


\section{LI, BEILHARZ, AND THORODDSEN}

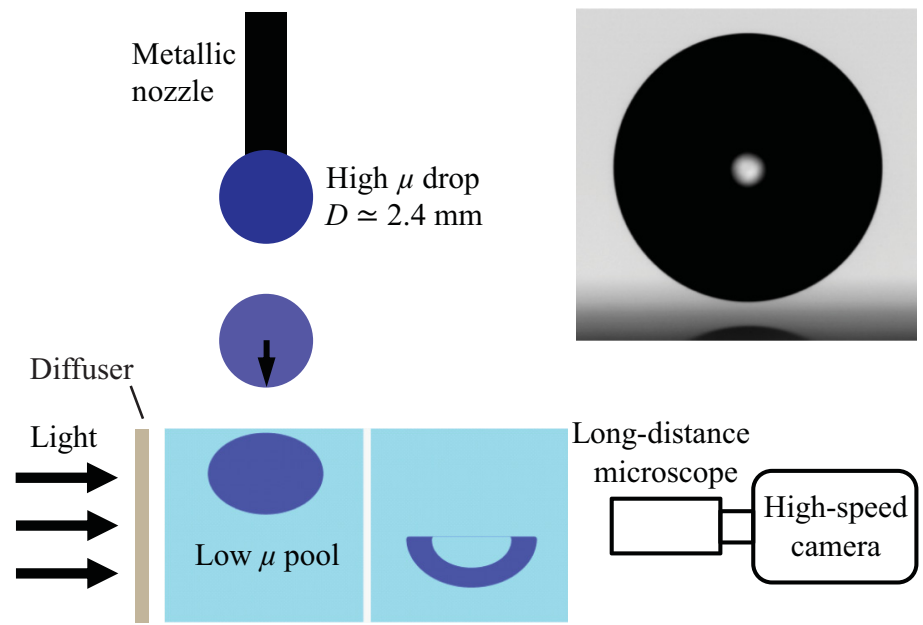

FIG. 2. Sketch of the experimental configuration, showing the metal tube used to pinch off the drop. The drop liquid is more viscous than the pool. Inset: The shape of the 100-cSt silicone-oil drop, after falling $18 \mathrm{~mm}$ from the nozzle. The drop is quite spherical with the aspect ratio $D_{v} / D_{H} \simeq 0.99$.

camera, with $1280 \times 800$ full-frame pixel area. A 350-W metal-halide lamp (Sumita Optical Glass, Inc.) combined with a diffuser provides enough backlighting to record with frame rates up to $30 \mathrm{kfps}$, even at the largest optical magnifications.

We use different but miscible silicone oils for the drop and the pool, with a much higher viscosity of the drop. On the other hand, the pool liquid viscosity was varied over the values of $0.65,1,1.5$, 2,5 , or $10 \mathrm{cSt}$. The properties of the different viscosity silicone oils are listed in Table I. The impact velocity $U$ was varied by changing the impact height between 20 and $500 \mathrm{~mm}$. Compared to free fall, the drop velocity is slowed down by the viscous pinch-off from the nozzle and was therefore measured in the free fall using the high-speed video camera.

The impact dynamics are characterized by the values of the Reynolds and Weber numbers

$$
\operatorname{Re}_{p}=\frac{\rho_{p} D U}{\mu_{p}}, \quad \mathrm{We}_{p}=\frac{\rho_{p} D U^{2}}{\sigma_{a p}},
$$

where $D$ and $U$ are the drop diameter and impact velocity, $\rho_{p}$ and $\mu_{p}$ are respectively the density and the dynamic viscosity of the pool, while $\sigma_{a p}$ is the surface tension of the pool liquid against the air.

TABLE I. Properties of the different liquids used in the experiments. Properties for silicone oils are taken from the manufacturers.

\begin{tabular}{lccccc}
\hline \hline Liquid & $\begin{array}{c}\text { Density } \\
\rho\left[\mathrm{g} / \mathrm{cm}^{3}\right]\end{array}$ & $\begin{array}{c}\text { Viscosity } \\
\mu[\mathrm{cP}]\end{array}$ & $\begin{array}{c}\text { Surface tension } \\
\sigma[\mathrm{dyn} / \mathrm{cm}]\end{array}$ & $\begin{array}{c}\text { Cap. length } \\
a[\mathrm{~mm}]\end{array}$ & Manufacturer \\
\hline Silicone oil 0.65 & 0.760 & 0.494 & 15.9 & 1.46 & Sigma-Aldrich Co. LLC \\
Silicone oil 1 & 0.818 & 0.818 & 16.9 & 1.45 & Clearco Products Co., Inc. \\
Silicone oil 1.5 & 0.851 & 1.28 & 17.9 & 1.46 & Clearco Products Co., Inc. \\
Silicone oil 2 & 0.880 & 1.76 & 18.2 & 1.46 & Clearco Products Co., Inc. \\
Silicone oil 5 & 0.915 & 4.57 & 19.7 & 1.48 & Clearco Products Co., Inc. \\
Silicone oil 10 & 0.935 & 9.35 & 20.1 & 1.48 & Sigma-Aldrich Co. LLC \\
Silicone oil 100 & 0.965 & 96.5 & 20.9 & 1.49 & Sigma-Aldrich Co. LLC \\
Silicone oil 500 & 0.970 & 485 & 21.2 & 1.49 & Sigma-Aldrich Co. LLC \\
\hline \hline
\end{tabular}




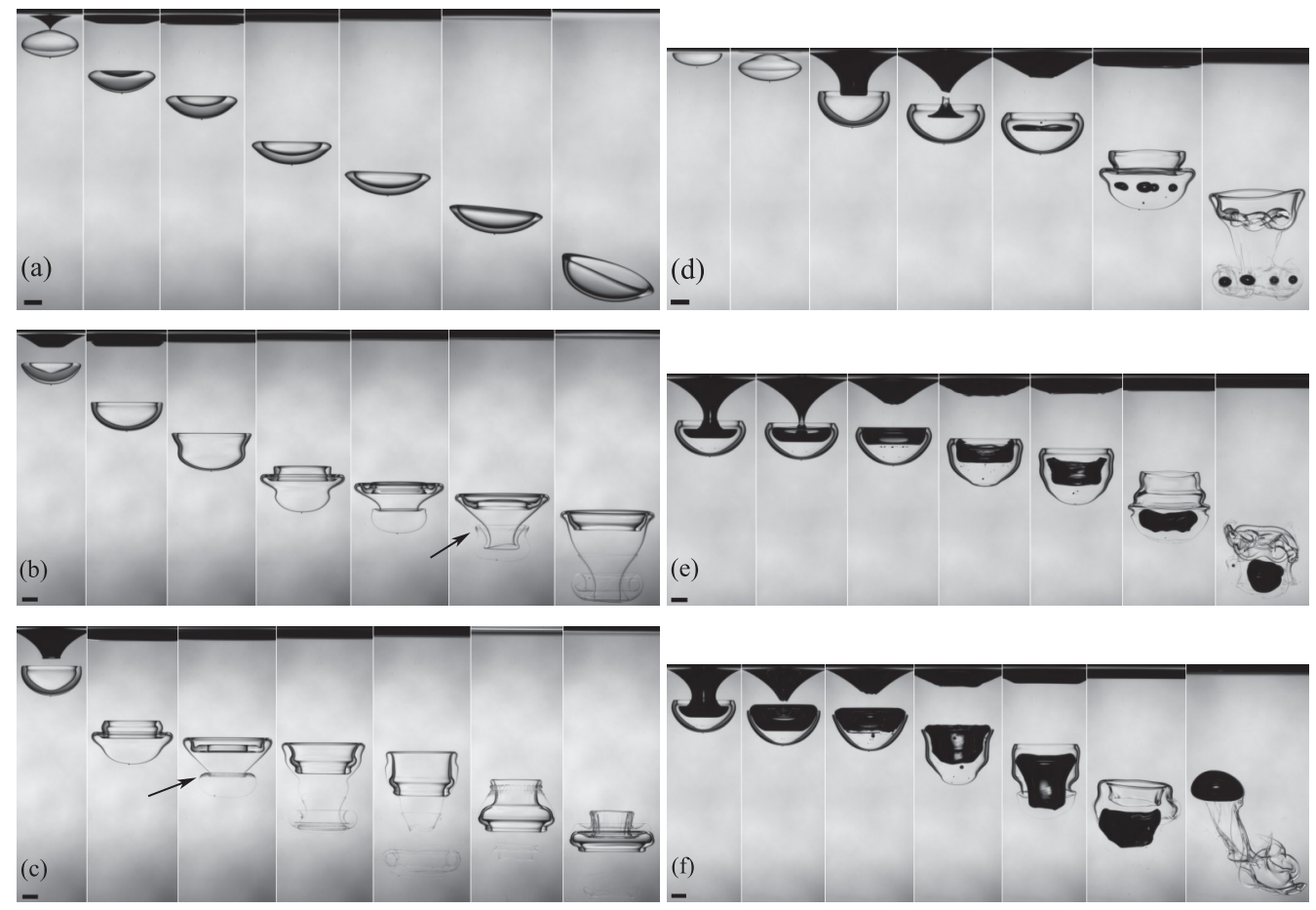

FIG. 3. Sequence of video images showing the effects of increasing the impact velocity, for a 500-cSt drop impacting a 0.65 -cSt pool. (a) $U=1.06 \mathrm{~m} / \mathrm{s}\left(\operatorname{Re}_{p}=3950, \mathrm{We}_{p}=130\right)$; (b) $U=1.62 \mathrm{~m} / \mathrm{s}\left(\operatorname{Re}_{p}=6045\right.$, $\left.\mathrm{We}_{p}=304\right)$; (c) $U=2.05 \mathrm{~m} / \mathrm{s}\left(\operatorname{Re}_{p}=7610, \mathrm{We}_{p}=482\right)$; (d) $U=2.20 \mathrm{~m} / \mathrm{s}\left(\operatorname{Re}_{p}=8206, \mathrm{We}_{p}=560\right)$; (e) $U=2.35 \mathrm{~m} / \mathrm{s}\left(\operatorname{Re}_{p}=8721, \mathrm{We}_{p}=634\right)$; and (f) $U=2.59 \mathrm{~m} / \mathrm{s}\left(\operatorname{Re}_{p}=9654, \mathrm{We}_{p}=778\right)$. Scale bars are all $1 \mathrm{~mm}$ long. See also video clips in the Supplemental Material [43].

The range of parameter values cover $1145<\mathrm{Re}_{p}<8500$ and $26<\mathrm{We}_{p}<600$. These parameters can also be defined with the drop properties, which gives $\operatorname{Re}_{d}$ two orders of magnitude smaller, with values of $\mathrm{Re}_{d}<15$, for the 500-cSt drop, while the $\mathrm{We}_{d}$ is essentially unchanged. For the 100-cSt drop in Sec. IV, $\operatorname{Re}_{d}<60$.

The resulting flow fields are all laminar and repeatable, except when large bubbles are entrained and disrupt the drop, as will be shown in Figs. 3(d)-3(f).

The rich space of observed phenomena arises not only from the impact $\mathrm{Re}$ and We but is fundamentally influenced by the difference in liquid properties between the drop and pool, with the viscosity ratio $\alpha=\mu_{d} / \mu_{p}$ and also the density difference $\Delta \rho=\rho_{d}-\rho_{p}$ being of importance. The viscosity ratio $\alpha$ is always kept large, mostly $>100$, while $\Delta \rho \leqslant 210 \mathrm{~kg} / \mathrm{m}^{3}$. The relative importance of the impact momentum and the buoyancy is characterized by two Froude numbers,

$$
\mathrm{Fr}_{\mathrm{air}}=\frac{U}{\sqrt{g D}}, \quad \mathrm{Fr}_{\mathrm{pool}}=\frac{U_{\text {pool }}}{\sqrt{g^{\prime} D}}
$$

where $U_{\text {pool }}$ is the sinking velocity inside the pool and the reduced gravity $g^{\prime}=g \Delta \rho / \rho_{p}$. Over the full range of impact velocities, we have $2<\mathrm{Fr}_{\text {air }}<21$, whereas for the cases with the vortex ring, the ranges are $9<\mathrm{Fr}_{\text {air }}<13$ and $14<\mathrm{Fr}_{\text {pool }}<24$. While the differential weight of the two liquids is important, this shows that the advection and wrapping due to the vortex ring are the primary drivers of the shape evolution, not localized buoyancy. On the scale of the sheet thickness, the influence of reduced buoyancy becomes negligible. This is not true for the top part of the viscous bowl [Figs. 3(b) and 3(c)], which is thicker and moves much more slowly. 


\section{LI, BEILHARZ, AND THORODDSEN}

\section{B. Particle image velocimetry}

In some experiments, the pool liquid was seeded with $6-\mu \mathrm{m}$ polymer microspheres (density $=$ $1.05 \mathrm{~g} / \mathrm{cm}^{3}$ ), which allowed us to perform particle image velocimetry (PIV) to estimate the velocity and vorticity fields, as presented in Sec. III F. We use backlit particle shadows [42] in combination with the narrow depth of focus of the long-distance microscope to confine the in-focus particle images to a narrow plane in the flow. At the largest magnifications, the depth of focus is measured to be only $180 \mu \mathrm{m}$, thus allowing us to measure the velocity in a vertical plane containing the axis of symmetry. The particles remain in focus over an extended distance of travel, demonstrating that our image plane passes close to the axis of symmetry. Using a high-speed video camera at frame rates up to $24 \mathrm{kfps}$, we can obtain time-resolved evolution of the velocity field. We use the Davis program from LaVision for the PIV calculations. Using interrogation window size of $16 \times 16 \mathrm{px}$ and $75 \%$ overlap, we achieve spatial resolution in the velocity field down to $26 \mu \mathrm{m}$, which is needed to accurately capture the details of the vorticity field.

\section{RESULTS FOR 500 CST DROP}

\section{A. Overall deformations and penetration into pool}

Figure 1 shows two typical examples of the buckled shapes of the stretched-out sheets of drop liquid, which are the focus of our study. We investigate this phenomenon over a range of liquid properties and impact velocities and identify the underlying dynamics, which we find to be controlled by a vortex ring. We see both axisymmetric and azimuthal buckling of the viscous sheets. The axisymmetry of the flow-geometry promotes azimuthal buckling when the viscous sheet is driven radially toward the axis of symmetry, at sufficient speed. Figure 4 highlights the region of parameter space where these buckling shapes occur. The filled symbols indicate prominent axial buckling, like that shown in Fig. 1(a). The viscous sheets are greatly stretched, thus becoming very thin. Therefore, buckling often occurs on very small length scales. The trade-off in the imaging, between spatial resolution and size of the field of view, requires taking numerous localized video sequences for identical impact conditions to observe the details of the buckling. The dynamics are repeatable and

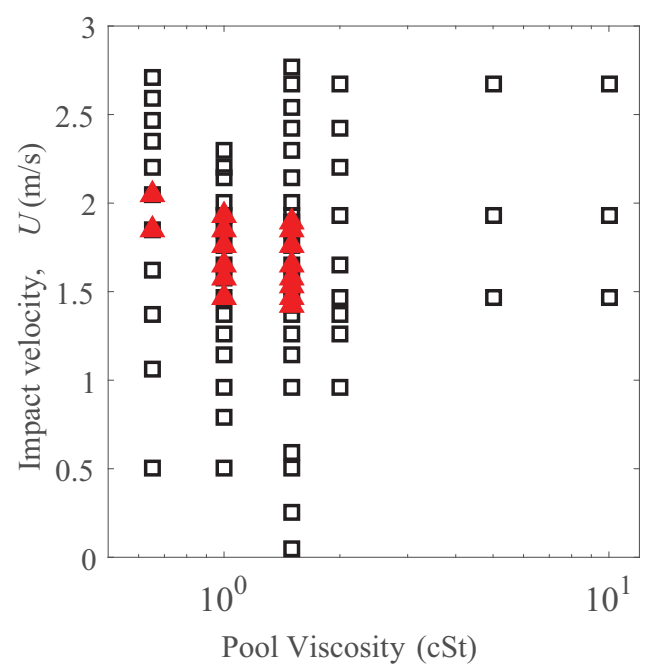

FIG. 4. Parameter space tested in the experiments (squares), for a fixed drop viscosity of $500 \mathrm{cSt}$ and $D=2.42 \mathrm{~mm}$. Prominent axisymmetric buckles (Fig. 1) occur under conditions marked by the red filled triangular symbols. 
do not change significantly during many subsequent impacts, as the drop liquid sinks to the bottom of the tank. This was verified by comparing with impacts on fresh pools, as was also done in Ref. [40].

Figure 3 shows the changes in the evolution of the drop shape, as the impact velocity is increased, while keeping the same drop and pool viscosities of 500 and $0.65 \mathrm{cSt}$ respectively. For the smallest $U$, Fig. 3(a), the drop deforms into a regular oblate shape, which separates from the pool surface and sinks slowly, taking the shape of a thick frisbee, then forming a shallow bowl shape, before tilting and breaking the symmetry in the last panel. For intermediate $U$, in Figs. 3(b) and 3(c), a vortex ring forms, which stretches and wraps thin sheets of the drop liquid around it. Weak indications of buckling are seen but are only visible as circumferential dark rings, which are marked with arrows. This buckling only becomes clear in close-up images, shown in Sec, III B. The intricate viscous sheet structure, which we focus on herein, occurs between $U \sim 1.4-2.0 \mathrm{~m} / \mathrm{s}$, for the 1-cSt pool, while at higher impact velocities, the air crater is pinched off and interferes with the vortex structure, as shown in Figs. 3(d)-3(f). In Fig. 3(d), an air torus is entrapped and subsequently breaks by capillary-driven Rayleigh instability into a few bubbles along the periphery. This breakup should disrupt any vortex ring, which may be present, but Fig. 3(d) shows an irregular vortex pulling the bubbles downward, against buoyancy. This indicates a sufficiently strong circulation and pressure gradient toward the core to trap the bubbles there. Then, for the highest impact velocities, in Figs. 3(c) and 3(f), the air crater grows in size and a larger bubble is pinched off. This bubble entangles with the drop liquid, producing irregular tendrils, as the bubble is so large that it reverses direction and rises back to the free surface by buoyancy.

In Fig. 5, we track the penetration depth of the drop liquid into the pool vs time, where we follow the deepest layer of the drop liquid. However, keep in mind that the most stretched thin sheets near the leading edge become almost imperceptible, further into the pool, making them difficult to track. In Fig. 5(a), we show this penetration for a wide range of impact velocities. It is clear that there is not a trivial relationship between the impact velocity $U$ and the penetration speed of the drop into the pool, as the penetration is affected by impact momentum, gravity through the air-crater formation, as well as the weight of the drop, which is of slightly larger density than the pool. For very low impact velocities, a stable air film can act as a trampoline, causing the drop to rebound from the surface, as was previously studied for these same liquids $[40,44]$. For the lowest velocities herein, the drop penetrates with a small crater [Fig. 3(a)] and its speed is driven both by impact momentum and gravity. Figure 6(a) shows another case of these dynamics. On the other hand, for the largest $U$, as shown in Fig. 6(b), a large crater forms and is pinched off. Here the crater breaks up into two large bubbles, which rapidly decelerate and deform the droplet. The curves in Fig. 5(a) for these two cases cross over. This shows that after the initial inertia-dominated phase, the penetration speed becomes larger for the much smaller value of $U$. In terms of the Froude number of the impact, the case with the largest $\mathrm{Fr}_{\text {air }}=21$ ends up with the lowest sinking velocity, $U_{\text {pool }} \simeq 0.040 \mathrm{~m} / \mathrm{s}$, as it has produced the largest crater. For the lower impact velocities, the fastest penetration would be possible with the smallest crater. The ideal case is if pool ejecta moves up along the drop surface to fully encompass it, without a crater. This has indeed been realized for impacts of viscous drops into low-viscosity pools [45], which leads the formation of high-speed apex jets. In Fig. 5(a), the oblate drop slows down, approaching a sinking velocity of $U_{p} \simeq 0.070 \mathrm{~m} / \mathrm{s}$, while the more deformed bowl-shaped drop for $U=3.24 \mathrm{~m} / \mathrm{s}$ has only $U_{p} \simeq 0.040 \mathrm{~m} / \mathrm{s}$ in the latter half of the figure. For reference, the terminal velocity of a spherical drop with our $D=2.4 \mathrm{~mm}$ is $\sim 0.132,0.091$, and $0.074 \mathrm{~m} / \mathrm{s}$, for the $0.65-, 1.0-$, and 1.5-cSt pools, respectively. The corresponding Reynolds numbers are 490, 220, and 120. The deformation of the drops into flatter shapes will certainly increase the form drag and lower the terminal velocities, closer to the observed values, but the sinking still occurs above the viscous-dominated Re regime. The inset of Fig. 6(a) shows the recirculating wake behind a descending flat drop, where the diffuse boundary layer moving up along the drop has introduced slight differences in the index of refraction.

For intermediate impact velocities, in Fig. 5(a), the penetration into the pool is faster, being a combination of the impact momentum, self-induced vortex motion, and buoyancy. There are two clear regimes for the penetration velocity in Fig. 5(b). First, the initial fast impact-controlled 


\section{LI, BEILHARZ, AND THORODDSEN}
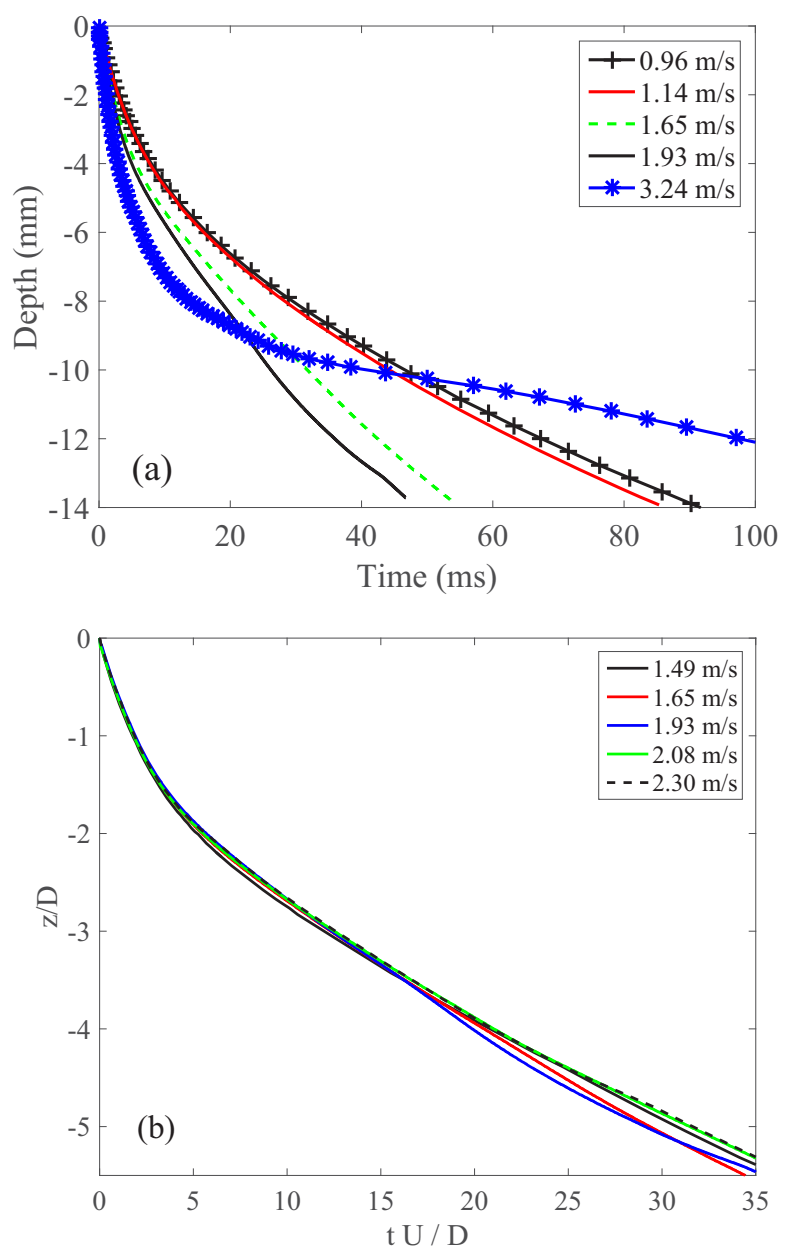

FIG. 5. The penetration depth of the bottom tip of the 500-cSt drop impacting 1-cSt pool liquid vs time. (a) Shown over the full range of impact velocities, $U=0.96,1.14,1.65,1.93$, and $3.24 \mathrm{~m} / \mathrm{s}$. (b) Drop-bottom trajectories for intermediate velocities, when the time is normalized by impact time and depth by the drop diameter. Results shown for $U=1.49,1.65,1.93,2.08$, and $2.30 \mathrm{~m} / \mathrm{s}$.

penetration, followed subsequently by the much slower buoyancy- and vortex-ring-driven motions. The initial fast regime lasts for about the first $5 \mathrm{~mm}$ of penetration, corresponding to about 2 drop diameters $D$. Here we have scaled the time with the impact time $D / U$. The initial penetration velocity is expected to be about half the drop impact velocity, owing to the additional virtual mass from the pool liquid; see Hendrix et al. [46]. Indeed, for the largest impact velocity $U$, the initial asymptotes are in reasonable agreement with this, when we include the difference in the drop vs pool densities. This gives $U_{p} \simeq(0.54 \pm 0.02) \times\left(\rho_{d} / \rho_{p}\right) U$ for both pool viscosities 0.65 and $1.0 \mathrm{cSt}$. In Fig. 5(b), the transition from the initial impact-dominated regime to a slower constant penetration speed occurs at $t \simeq 4 D / U$. In the second regime, we see very similar trajectories over this range of intermediate impact velocities. The slightly faster normalized speeds occur for the most prominent vortex rings for $U=1.65$ and $1.93 \mathrm{~m} / \mathrm{s}$, as will be studied with the PIV measurements.

It is nontrivial to disentangle the contributions from the initial impact momentum and the vortexinduced translation, whereas the buoyancy is clearly not as important when the drop liquid has been stretched into fine sheets. See for example, the sequence of images in Fig. 3(c) (and later Fig. 17), 

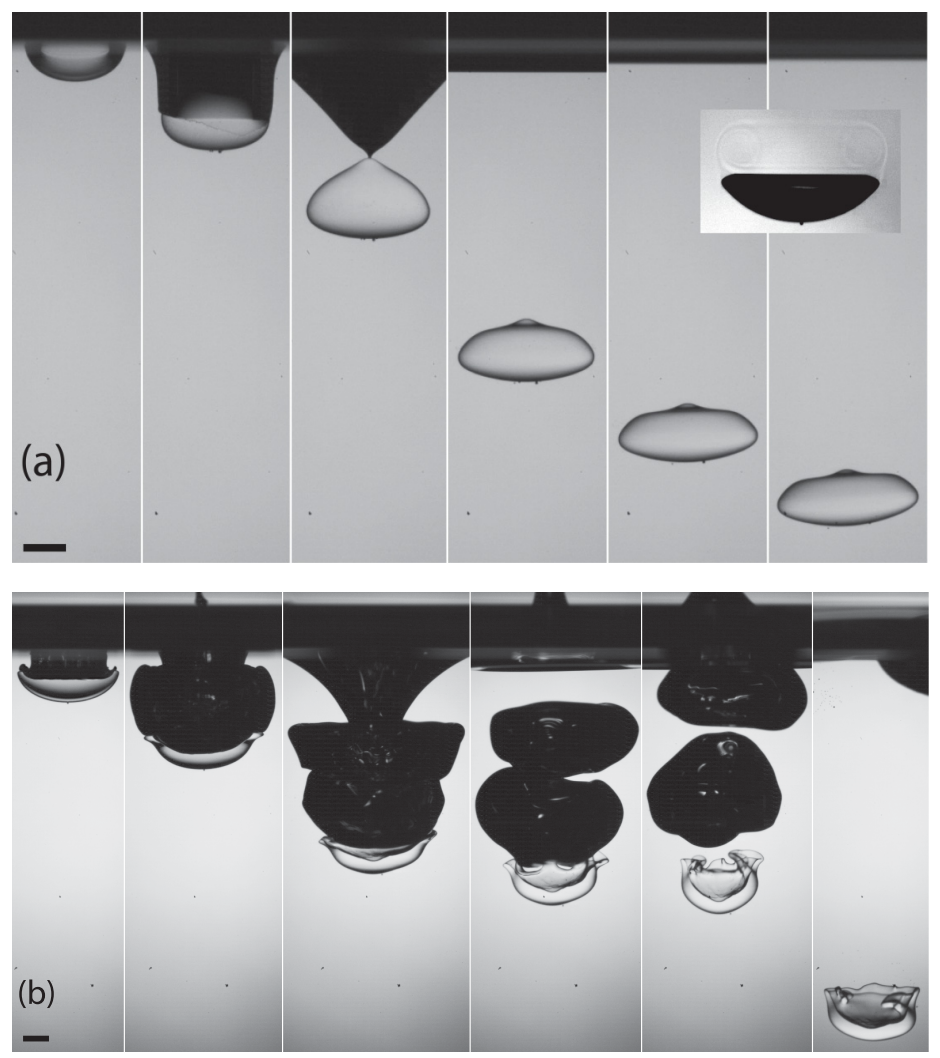

FIG. 6. Comparison of low- and high-speed impacts of a 500-cSt drop onto (a) a 1.5-cSt pool liquid, at $U=$ $0.73 \mathrm{~m} / \mathrm{s}\left(\operatorname{Re}_{p}=1180, \mathrm{We}_{p}=62, \mathrm{Fr}_{\mathrm{air}}=4.7\right)$. Times shown are at $t=1.5,4.9,11.4,34.1,56.8$, and $79.5 \mathrm{~ms}$ after first contact. The inset shows a recirculating vortical region in the wake of the drop, for a similar setup with 1-cSt pool and $U=1.14 \mathrm{~m} / \mathrm{s}$. (b) 500-cSt drop impacting 1-cSt pool at $3.24 \mathrm{~m} / \mathrm{s}\left(\operatorname{Re}_{p}=7840, \mathrm{We}_{p}=\right.$ $1220, \mathrm{Fr}_{\mathrm{air}}=21$ ), shown at $t=1.0,3.6,18.6,34.6,44.6$, and $167 \mathrm{~ms}$. Scale bars are $1 \mathrm{~mm}$.

where the top part of the bowl, which contains most of the drop mass, sinks much more slowly than the bottom part, which is driven downward by the vortex ring. For the same drop and pool liquids, drop-induced vortex rings are known to effectively transport oxygen-rich surface water into the bulk [47]. However, in our configuration, we will show in Sec. III F, the vortex ring must work against the viscous stresses in the drop and break through it, thereby reducing its strength.

\section{B. Axisymmetric buckling}

We now focus in on the buckled shapes, shown earlier in Fig. 1(a). Figures 3(b) and 3(c) show the overall drop-shape evolution and buckling. In both panel 4 of Fig. 3(b) and panel 2 of Fig. 3(c), the first stage of the buckling occurs in the upper part of the bowl-shaped drop liquid, where the edges fold inward. This is driven by the larger weight of the thicker top section but is also assisted by the vortex ring, which has entered the bowl, as will be shown in the PIV results. Then a second much finer buckling is observed along the center of the bowl, pointed out by the arrow in Figs. 3(b) and 3(c), where it is barely visible, as dark circumferential lines. Figure 7 presents an enlarged image sequence of this axisymmetric buckling. It shows clearly how the flow induced by an internal vortex ring compresses the upper edge of a larger fold in the sheet, producing a sequence of ever finer buckles. For buckling in the axisymmetric plane, these folds in the sheet are coherent around the entire periphery, thereby remaining axisymmetric, as is seen by the horizontal lines in Fig. 7. This is 

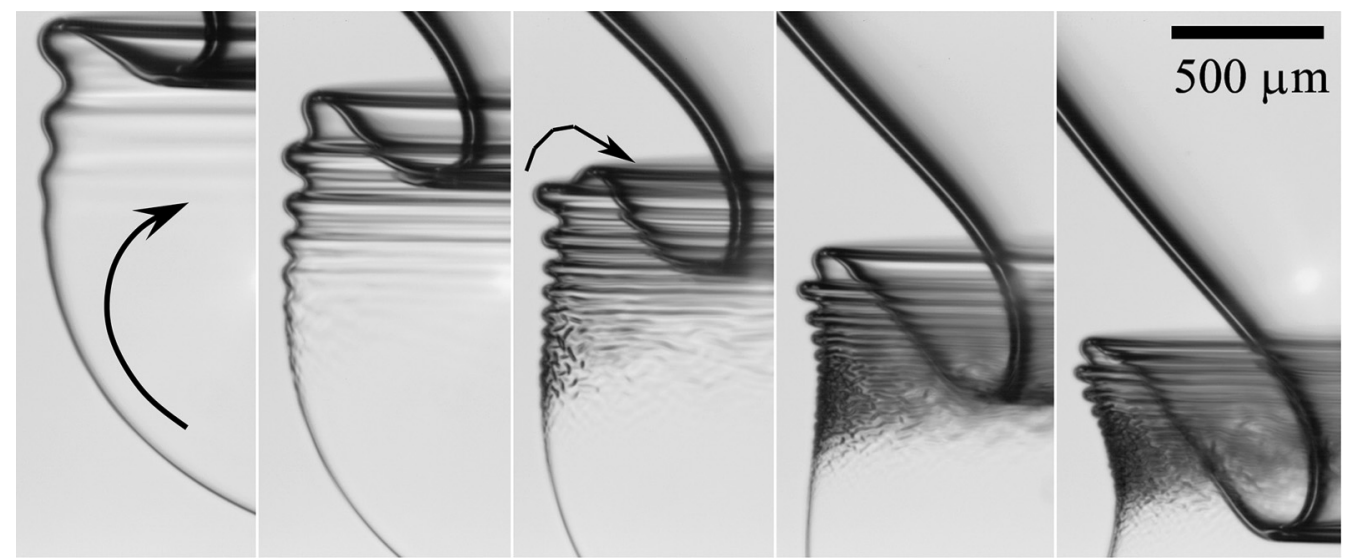

FIG. 7. Closeup of the axisymmetric buckling occurring in the region above the vortex ring, which is pointed out by the arrow in Fig. 3(b), for similar conditions, with 1-cSt pool. $U=1.65 \mathrm{~m} / \mathrm{s}, \operatorname{Re}_{p}=3993$, and $\mathrm{We}_{p}=319$. The panels have a relative timing of $t=0,2.1,4.0,6.7$, and $9.75 \mathrm{~ms}$. The arrow in the first panel shows the direction of the particle motions, relative to the sheet, which is induced by the vortex ring. The arrow in the third panel shows the unwinding of the buckles as they are advected around the large top fold. See also the video clip in the Supplemental Material [43].

true for the first five folds, but the approximately five following foldings are more random, showing a combination of axial and azimuthal ridges, often at $45^{\circ}$ angles. This is driven by compression of the sheet in both the axial and azimuthal directions. Bending, as the sheet goes from convex to concave, could possibly also play a role in the orientation of the buckles. In Fig. 7, the buckling starts near the top of the main bend, where the sheet is thickest, and then propagates downward, where the sheet is thinner. The wavelength of the buckles reduces accordingly. We can estimate the rate of compression from the video if we assume that the crests and troughs do not move as waves along the sheet but remain composed of the same fluid elements. These crests can be tracked, using image sequences like those in Fig. 8. This is presented in Fig. 9(a). Here the overall compression rate is about uniform, approximately $-200 \mathrm{~s}^{-1}$. The initial wavelength at the start of each buckle is shown in Fig. 9(b). Here we can ascertain the wavelength as a function of sheet thickness. The wavelength of the buckles $\lambda$ may be expected to be proportional to the local sheet thickness $\delta$ and

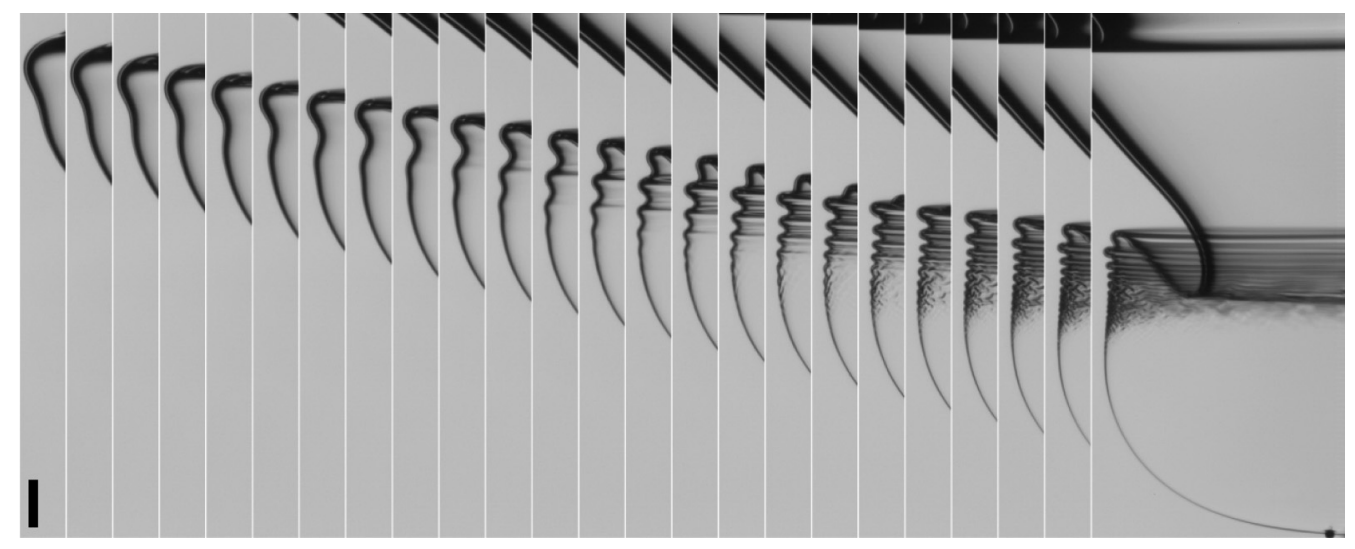

FIG. 8. Sequence of images showing the buckling, showing every 10th frame, i.e., spaced by $0.5 \mathrm{~ms} . U=$ $1.65 \mathrm{~m} / \mathrm{s}, \operatorname{Re}_{p}=3993$, and $\mathrm{We}_{p}=319$. Scale bar is $200 \mu \mathrm{m}$. 

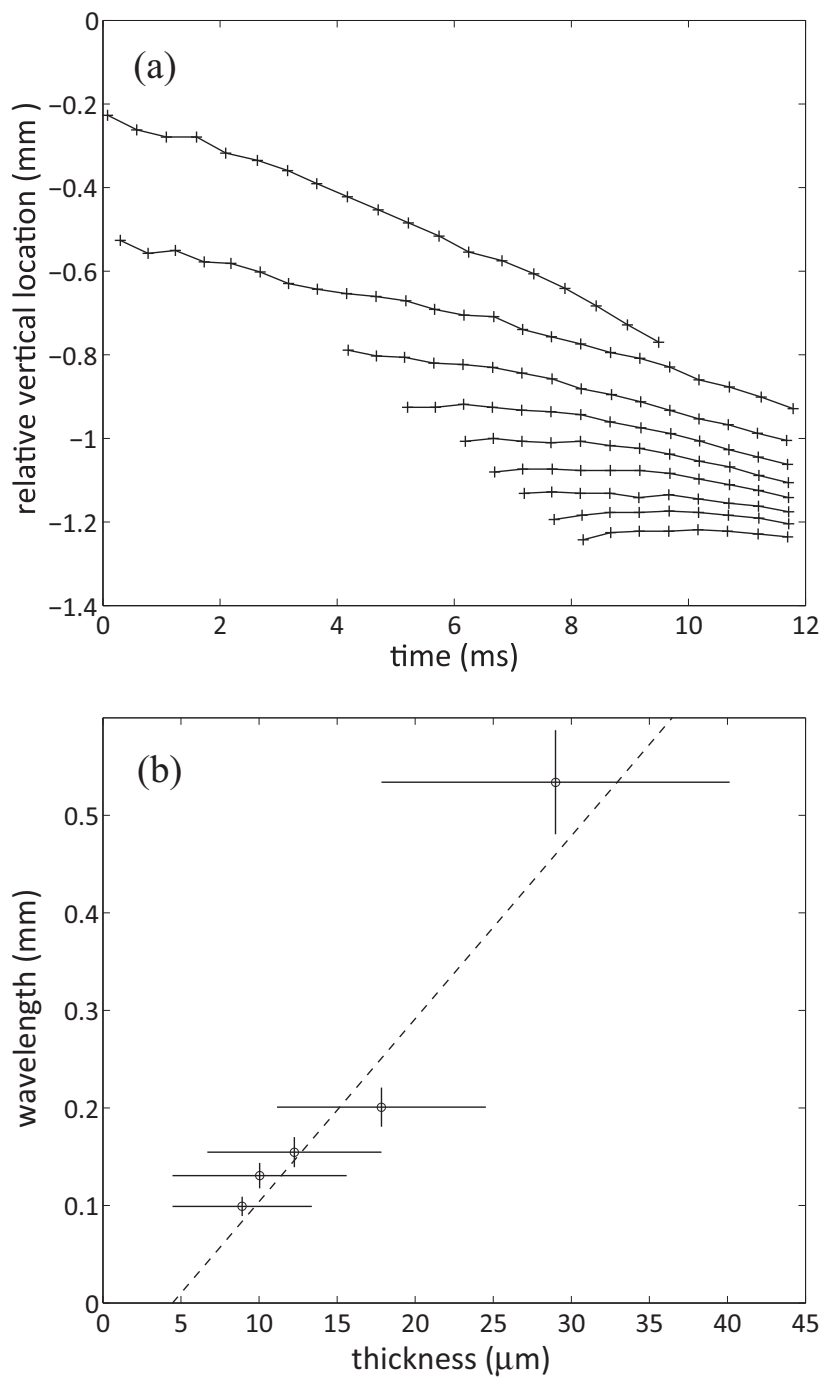

FIG. 9. (a) Tracing the location of the buckle crests vs time, for the conditions in Fig. 8. (b) The original wavelength of the buckles vs the average local sheet thickness approximated from direct side-view imaging.

the strength of its viscosity $\mu_{d}$. It is indeed shown in Fig. 9(b) that the wavelength of the buckling is approximately linear with the thickness of the sheet. Here $\lambda \simeq 19 \times \delta$. The large error bars come from the inaccuracy in determining the sheet thickness owing to refraction at the glancing view. On the other hand, we point out that the dependence on $\mu_{d}$ is not easily verified, as systematic changes in drop viscosity lead invariably to qualitative changes in the global flow field and sheet thickness. In other words, viscosity and compression rates cannot be independently varied.

The first bends of the sheet are in this case unbent, or straightened out, when they are pulled around the top of the larger bend and stretched downward, as is indicated by the curving arrow in the third panel in Fig. 7 (see also a video clip in the Supplemental Material [43]).

It is of interest to note the strong axisymmetry of the viscous sheets, in Figs. 3(a)-3(c), where the tip of the innermost winding is in perfect alignment between the left and right sides of the vortex. There is no obvious feedback between these two sides. Indeed, in later Figs. 12(a) and 16(a), the 


\section{LI, BEILHARZ, AND THORODDSEN}

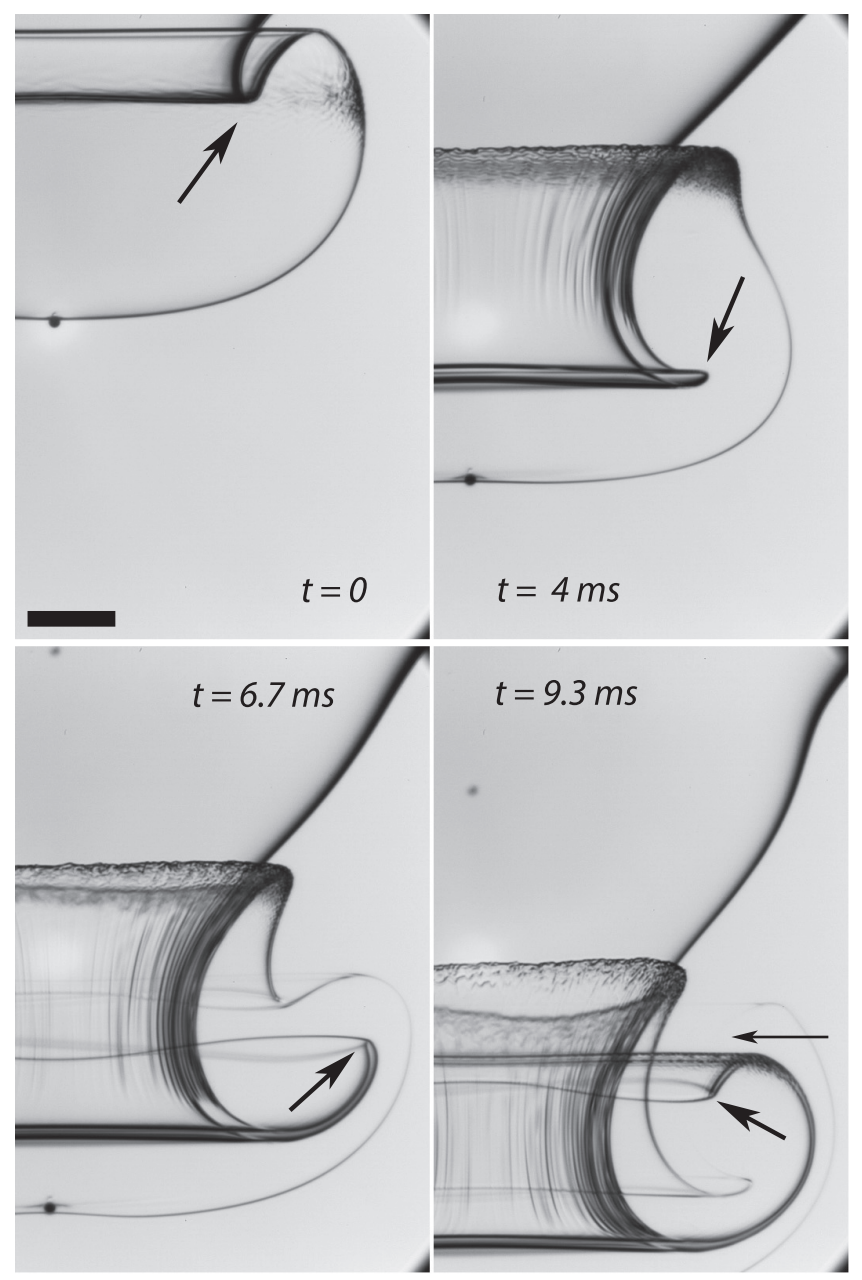

FIG. 10. Closeup showing how a fold of the thin sheet of drop liquid is pulled in and wrapped around the vortex. The arrows point at the tip of the first fold being pulled in. In the third panel, a second fold begins to be pulled in. Azimuthal buckling is also observed starting in the second panel. For 500-cSt drop into 0.65-cSt pool, from $H=220 \mathrm{~mm}, U=1.85 \mathrm{~m} / \mathrm{s}, \operatorname{Re}_{p}=6888, \mathrm{We}_{p}=395$. Scale bar is $500 \mu \mathrm{m}$ long.

location of the innermost wrinkled sheets are greatly out of phase along the azimuthal direction; see arrow in Fig. 16(a).

\section{Azimuthal buckling}

In some cases we observe azimuthal buckling, shown on right side of Fig. 1. This is manifest as vertical ridges in the images and arises when the primary compression is owing to flow-induced reduction of the radius of a cylindrical sheet. Keep in mind that the viscous sheets which move around the vortex core will be subjected to alternating azimuthal stretching and compression, as they are advected away from or toward the axis of symmetry.

Figure 10 shows such vertical streaks on the second to the innermost section. This figure also highlights the pulling in of a fold of the outer viscous sheet (see arrows) to form a double-walled sheet which is wrapped around the vortex. The third panel shows the beginning of the pulling of a second fold. This is even followed by the third fold (see video). The rate of compression can be 


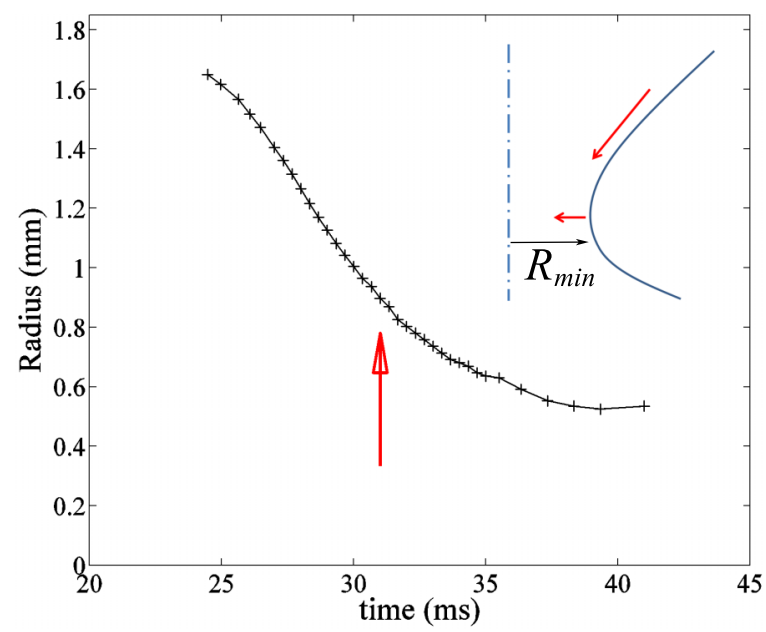

FIG. 11. The reduction with time of the minimum radius $R_{\min }$ of the inner cylinder (under the conditions in Fig. 10), leading to azimuthal buckling at the time of the red arrow.

approximated from the videos, as is done in Fig. 11, which shows the minimum radius $R_{\min }$ vs time, corresponding to the case in Fig. 10. Here we get a rate of

$$
\frac{1}{R_{\min }} \frac{d R_{\min }}{d t} \simeq 115 \mathrm{~s}^{-1}
$$

Typical azimuthal wavelengths are around $\lambda \simeq 66 \mu \mathrm{m}$ determined from the start of buckling in Fig. 10. In this case, the buckling appears to be in the second viscous sheet from the axis of symmetry. It is therefore the speed of the rotation of this layer around the vortex ring which determines the compression rate, not the minimum radius vs time which is plotted in Fig. 11. The inset in Fig. 11 explains this concept; i.e., the sheet can be compressed simply by the motion along its length (longer arrow) and does not require a reduction in $R_{\min }$. Indeed, the rotational velocity of the tip of this fold is measured at an average $u_{\theta} \simeq 320 \mathrm{~mm} / \mathrm{s}$, giving a compression rate, when the tip travels from the outer to inner edge of the torus (see thin arrow in Fig. 10), producing compression rate of $215 \mathrm{~s}^{-1}$, considerably larger than the value above obtained from Eq. (1) from the reduction in $R_{\min }$.

Figure 12 shows a set of three separate azimuthal buckling events, occurring at different depths along the cylindrical viscous sheet. Note that the two layers in the middle consist of double-walled viscous sheets. The buckled ridges are on the inner side of the vortex ring, while the outer side has stretched out the undulations and the sheets look smooth.

However, the azimuthal wavelengths are difficult to determine later on when multiple layers of buckles overlay in the image, like in Fig. 12. We can therefore not estimate $\lambda$ accurately, nor can we estimate thicknesses of these greatly stretched sheets.

It is interesting to contrast the azimuthal buckling in Fig. 12 with the axial buckling in Fig. 13. The latter one occurs when the viscous sheet approaches the stagnation region near the bottom stagnation point (when observed in the frame of reference of the vortex ring). The wavelength of these axial buckles at this late stage [arrows in Fig. 13(b)] is about constant of $\simeq 70 \mu \mathrm{m}$, but they are rather undulating in the circumferential direction. However, in this image the amplitude has saturated as the adjacent buckles touch. The wavelength at the onset of this buckling is $\lambda \simeq 150 \mu \mathrm{m}$.

Figure 14 shows buckling of the sheet well above the vortex ring, where the orientation of the ridges appears random. We speculate this is due to mixed-mode buckling, where both axial and azimuthal compression is at play. This is supported by the very slow reduction in the radius of the 


\section{LI, BEILHARZ, AND THORODDSEN}
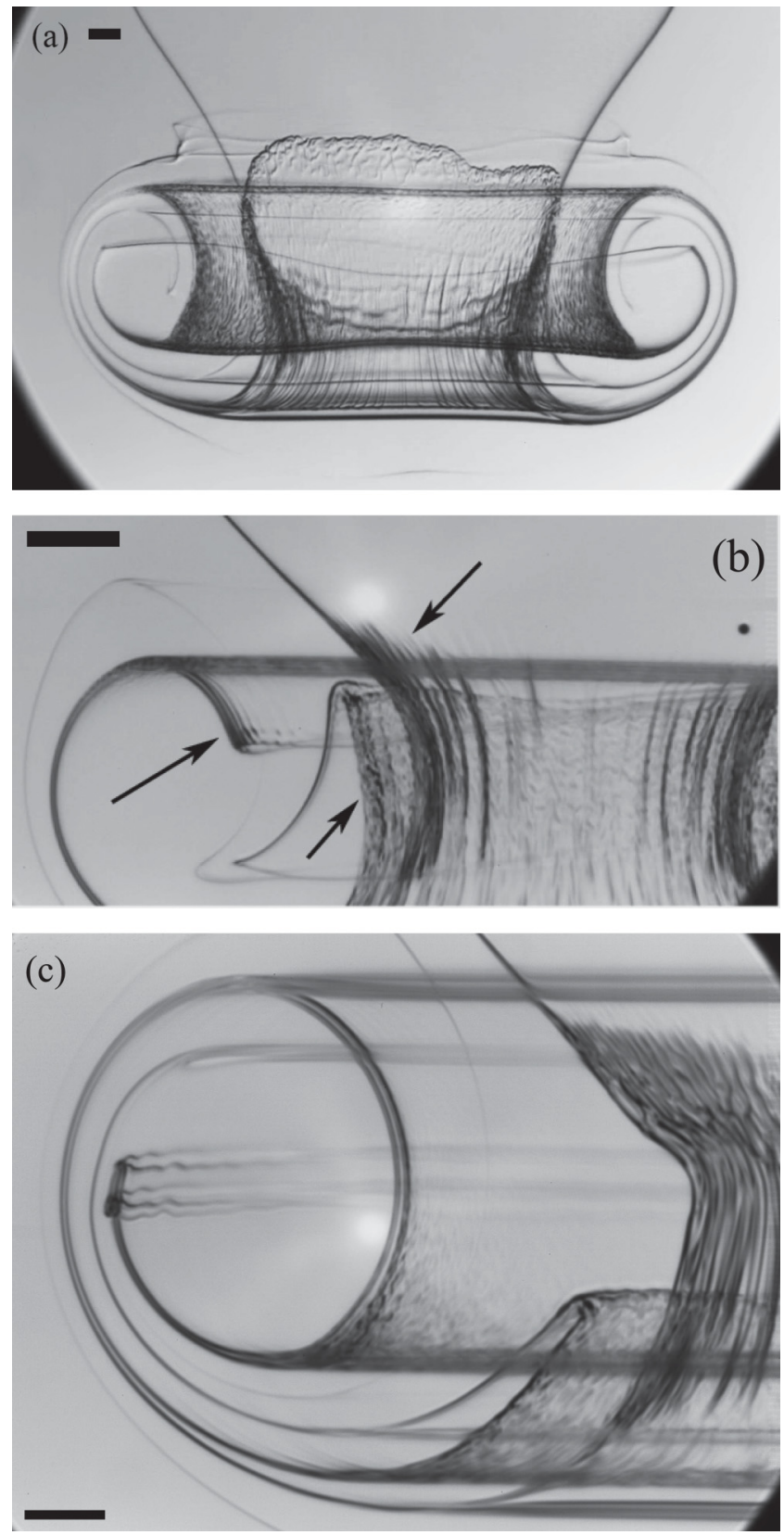

FIG. 12. Azimuthal buckling of a 500-cSt drop impacting a 0.65 -cSt pool, for $U=1.85 \mathrm{~m} / \mathrm{s}, \operatorname{Re}_{p}=6888$, $\mathrm{We}_{p}=395$. From the region marked with the arrow in Fig. 17 with a progressively larger optical magnifications. (a) The middle sheet does not seem to be buckled, whereas the other two are. (b) Buckling of three nested layers under compression. (c) Image with the largest magnification $(1.94 \mu \mathrm{m} / \mathrm{px})$. Note the four windings, three of which are double sheets while the outermost sheet is single. Scale bars are all $200 \mu \mathrm{m}$ long.

cylinder, at a rate of $(1 / R)(d R / d t) \simeq 15 s^{-1}$. This highlights the fact that the compression does not have to be unidirectional, as most theories assume [23].

Figure 15 shows continuous wrapping of the drop liquid around the vortex core, as the vortex ring travels further into the pool. 

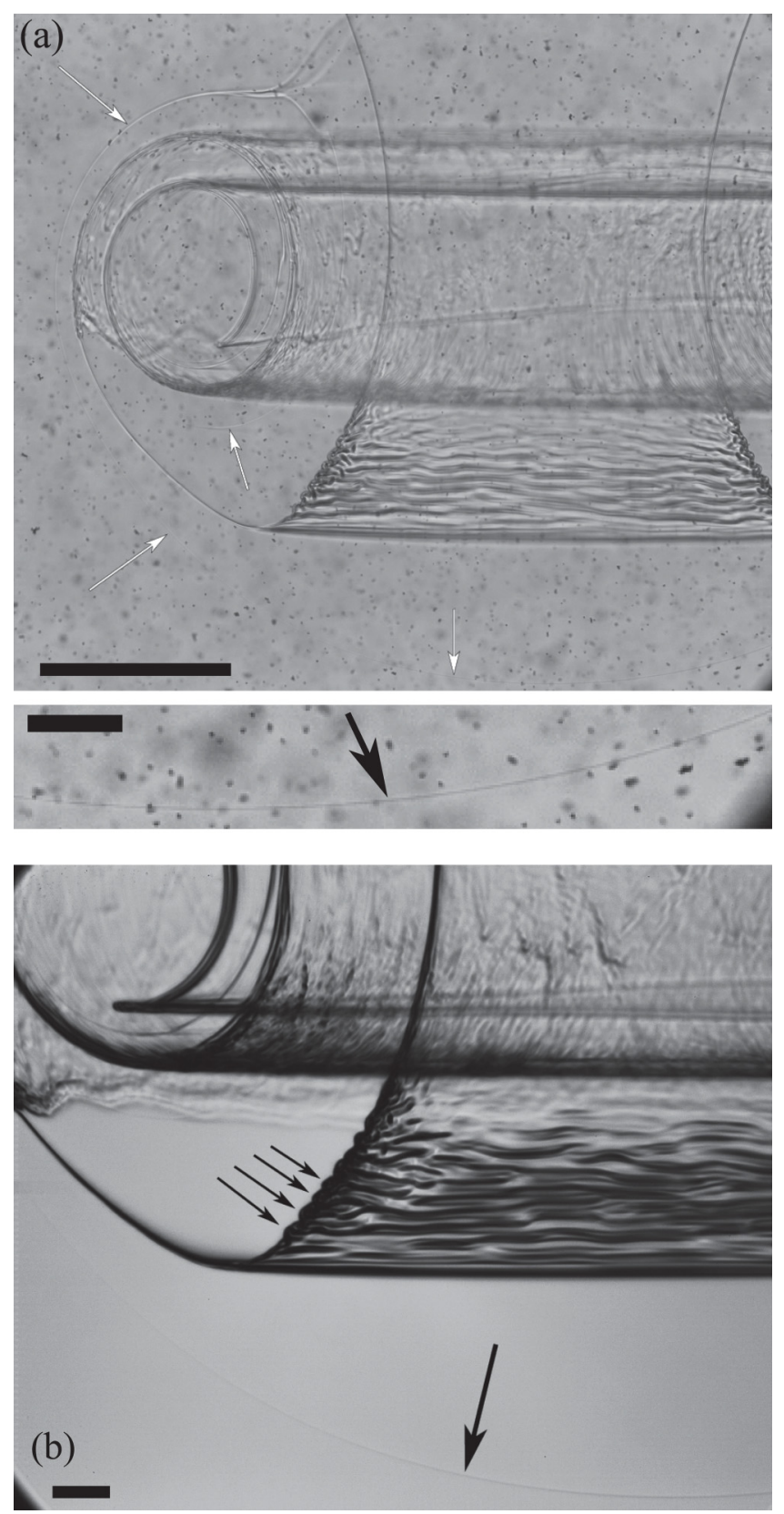

FIG. 13. (a) Closeup of the sheet wrapping by the vortex ring with axial buckling, as the sheet nears the bottom stagnation point, for $U=1.93 \mathrm{~mm} / \mathrm{s}, \operatorname{Re}_{p}=4671, \mathrm{We}_{p}=436$. The white arrows point out the bottom sheet which is smooth but stretched so thin that it is barely visible. In this realization, we have seeded the pool liquid with fine particles for PIV. The bottom image focuses in on a subsection of the finest thread above, which is less than one pixel wide $<3 \mu \mathrm{m}$. Top scale bar is $1 \mathrm{~mm}$ long, but the bottom one is $200 \mu \mathrm{m}$. (b) Closeup of the inner buckling and wrapping by the vortex ring, now without seeding particles. Bottom arrow points at the outermost viscous sheet. The image contrast has been greatly enhanced to make the fine sheet visible. Scale bar is $200 \mu \mathrm{m}$. 

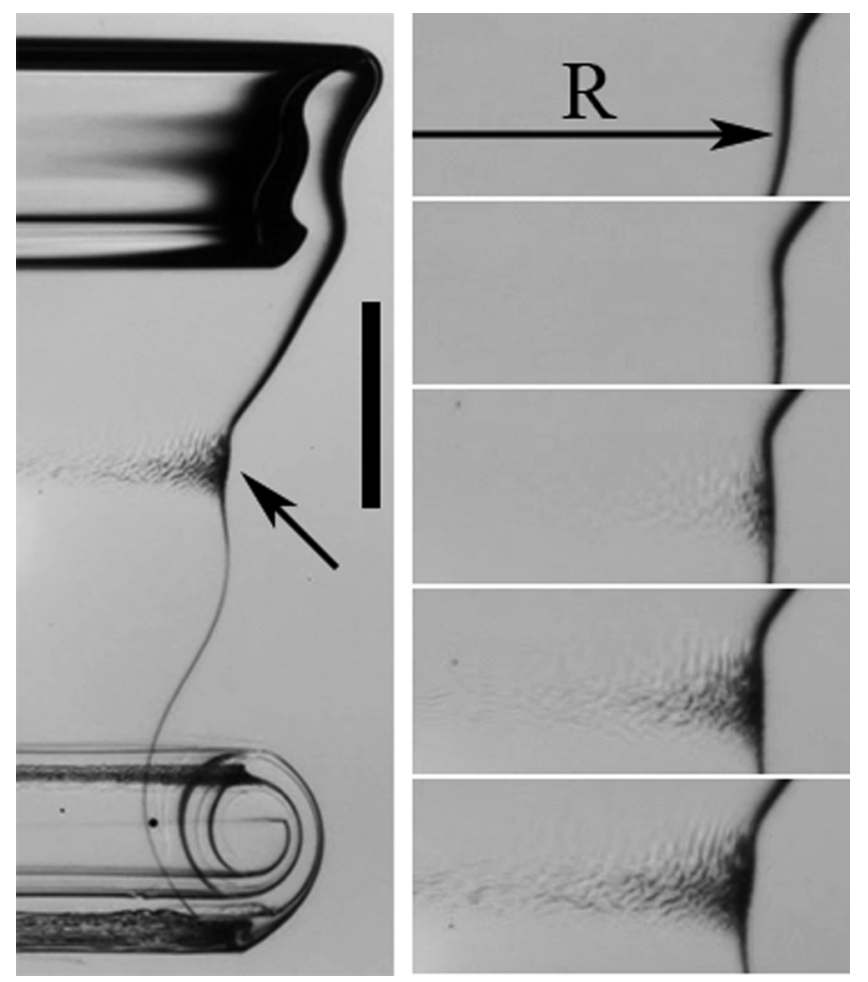

FIG. 14. Closeup of mixed-mode axial and azimuthal buckling, occurring along a cylindrical section of the viscous sheet, for $U=1.85 \mathrm{~m} / \mathrm{s}, \operatorname{Re}_{p}=6888, \mathrm{We}_{p}=395$. The frames on the right side are spaced by $2 \mathrm{~ms}$. The left side of the frames coincides with the axis of symmetry. Scale bar is $1 \mathrm{~mm}$ long.

\section{Surface area of drop-pool interface}

One measure of the stirring of the drop liquid inside the pool is the growth in the surface area between the two liquids. This area can in principle be measured accurately from the video sequences. However, the buckling makes this estimate uncertain and refraction inside the multiple windings also interferes. The axisymmetry assumption certainly underestimates the true area, when it contains azimuthal buckles. Furthermore, the original bottom sheet of drop liquid can become so greatly stretched that it disappears from our imaging. This is highlighted in Fig. 13, where we point out two such examples, using an image taken at the maximum magnification, where the sheet is just about the disappear. However, for up to three windings this area can be manually traced out accurately.

The arrows in Fig. 10 showed how some of the wrapped sheets of the drop liquid are double, i.e., composed of two sheets. This arises as a tongue of the outer sheet is pulled toward the centerline by the vortex. Keeping this in mind, we manually trace out the convoluted interface shapes, marking a continuous series of points along the entire interface; see Fig. 16(b). We then use the axisymmetry to calculate the total interface area, which is a fundamental quantity for determining the amount of molecular mixing between the two liquids. Figure 16(c) shows how the total area grows with time. Initially, the area grows slowly, as the drop deforms into the bowl shape. Then it grows much more rapidly, as the vortex ring wraps up the multiple sheets, by pulling in three double folds as shown in Figs. 10, 12, and 13. Here the interface area has grown $\sim 45$-fold larger within the first $50 \mathrm{~ms}$.

Knowing the area and volume of the drop liquid we can estimate the sheet thickness. However, a significant fraction of the drop volume is left behind in the thick collar. Therefore, only a small section of the area at the bottom of the bowl, marked by arrows in Fig. 17, is stretched around the 

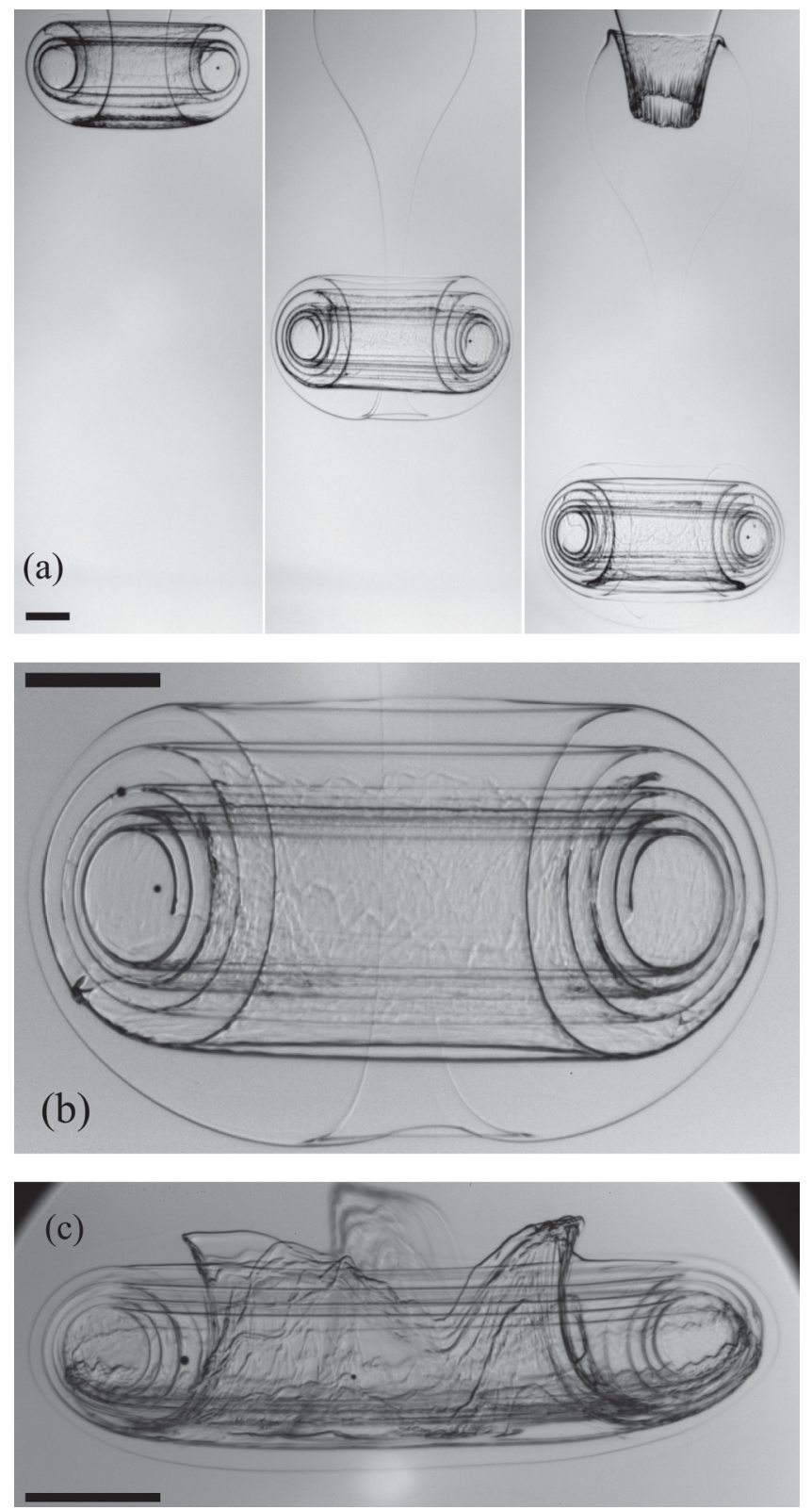

FIG. 15. (a) Six clearly visible wrappings around the vortex ring, further into the pool, for $v_{d}=500 \mathrm{cSt}$, $v_{p}=1 \mathrm{cSt}$, for $U=1.93 \mathrm{~mm} / \mathrm{s}, \operatorname{Re}_{p}=4671, \mathrm{We}_{p}=436$. The last frame shows a late-stage azimuthal buckling near the top. (b) Closeup of clearly visible wrappings around the vortex ring, for the same conditions as in panel (a). Image has been greatly enhanced. (c) The vortex ring further down in the pool, for $v_{d}=500 \mathrm{cSt}$, $v_{p}=0.65 \mathrm{cSt}, U=1.85 \mathrm{~m} / \mathrm{s}, \operatorname{Re}_{p}=6888, \mathrm{We}_{p}=395$, when the sheets have become quite irregular. The outermost viscous sheet has disappeared at this optical resolution. Scale bars are all $1 \mathrm{~mm}$ long.

vortex. For this particular case, we estimate that less than $15 \%$ of the volume is part of the stretched sheet. At this early time, we can estimate the local thickness of the sheet and integrated the volume assuming axisymmetry. The stretching is of course highly nonuniform along its length, as is indeed 


\section{LI, BEILHARZ, AND THORODDSEN}
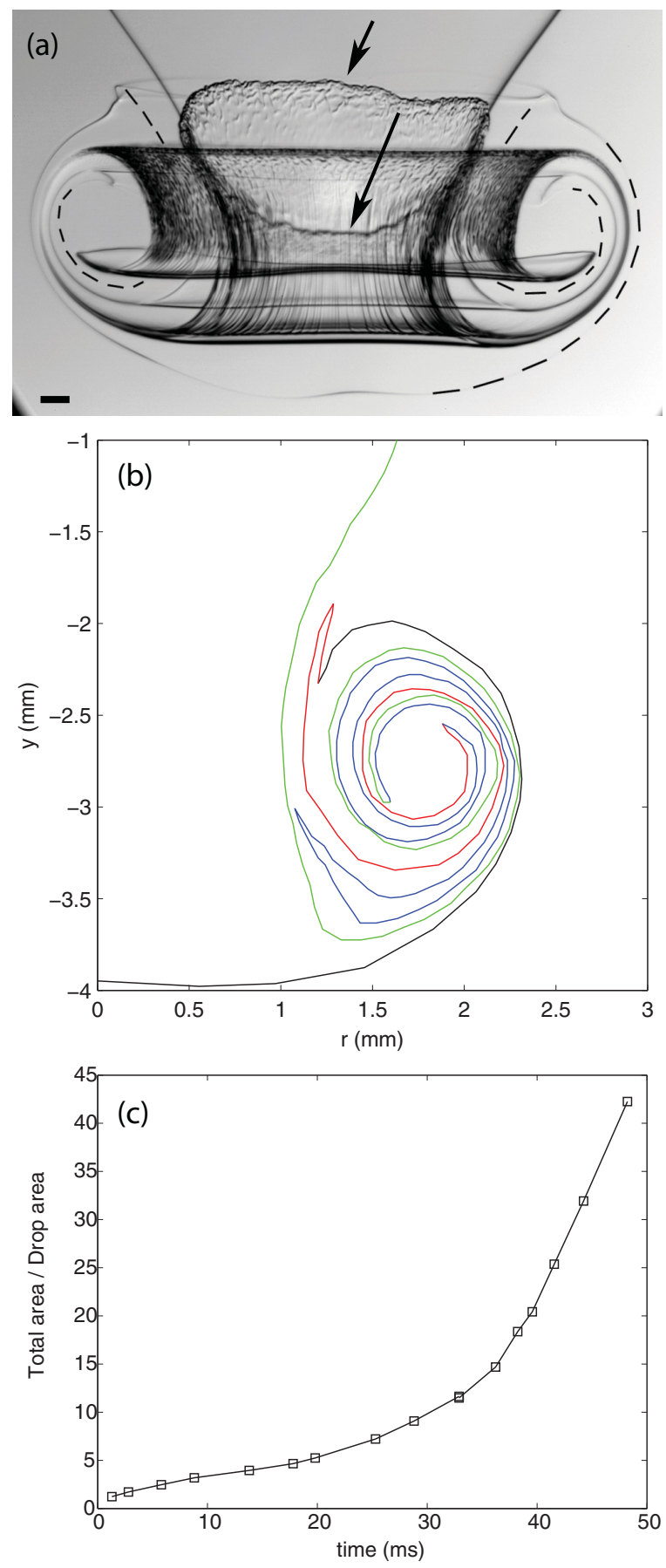

FIG. 16. (a) Image of the wrapped viscous sheets, where the thinnest sheet has been traced by dashed curves. (b) Manually traced instantaneous interface shown at a slightly later time than the above image. Taken from a different realization which allows a longer time series for measuring changes in the surface area, as shown in part (a). The line color has been changed between the tips of the three individual folds. (c) Total interface area normalized by the initial spherical drop area. Evaluated from two realizations, like those in panel (b), for 500-cSt drop and 0.65-cSt pool, impacting at $U=1.85 \mathrm{~m} / \mathrm{s}$, for $\operatorname{Re}_{p}=6888, \mathrm{We}_{p}=395$. 


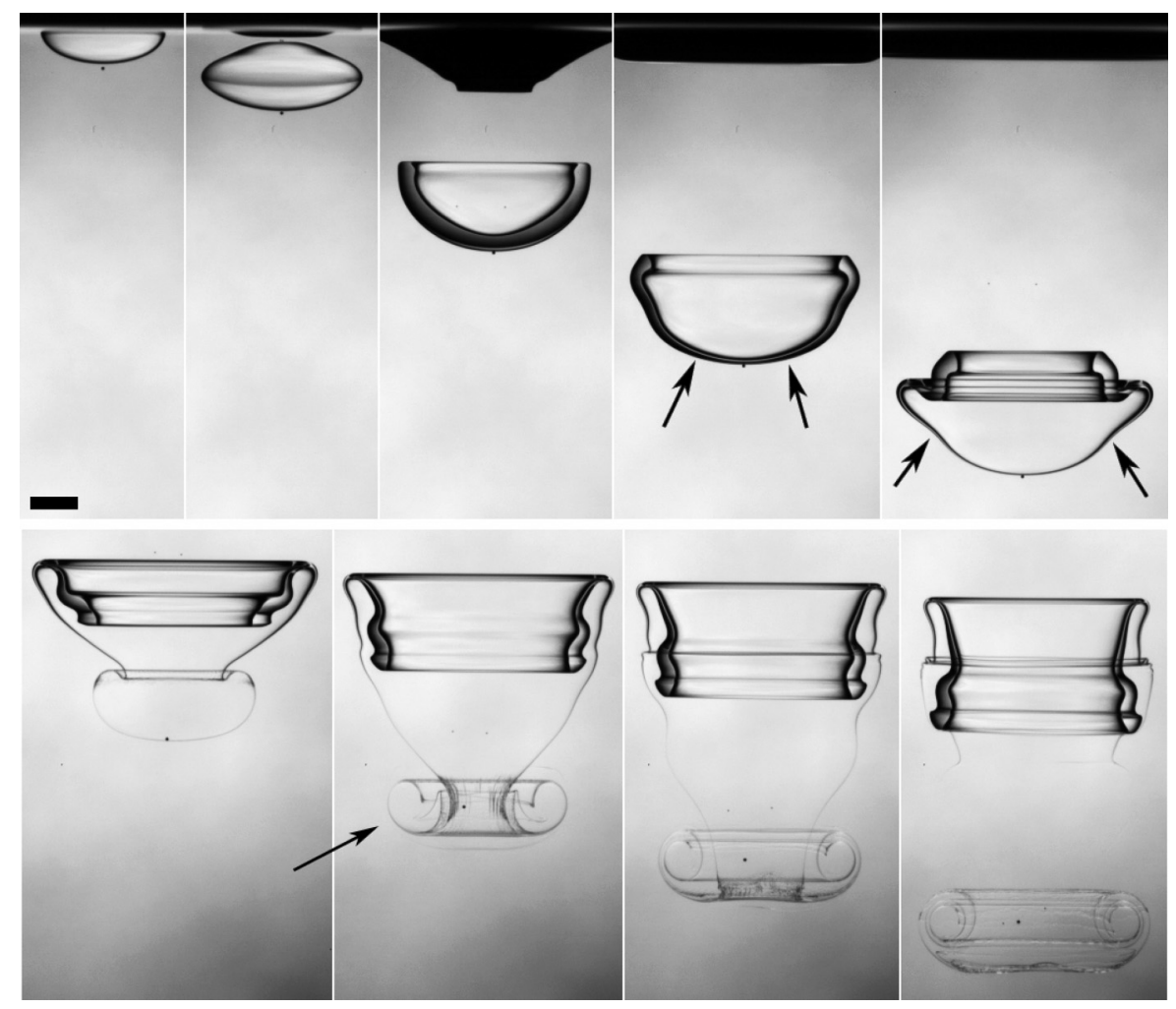

FIG. 17. Shape evolution and azimuthal buckling (bottom arrow) during the impact of a 500-cSt drop onto a 0.65 -cSt pool liquid, for $U=1.85 \mathrm{~m} / \mathrm{s}, \operatorname{Re}_{p}=6888, \mathrm{We}_{p}=395$. Times shown are, from left to right and top to bottom, at $0.47,1.37,5.8,13,22,30,40,47$, and $56 \mathrm{~ms}$ after first contact. Pairs of arrows point out which section of the drop liquid is stretched out by the vortex ring, i.e., $<15 \%$ of the drop volume. Scale bar is $1 \mathrm{~mm}$.

highlighted by the compressive sections manifest by the observed buckling. Subtracting the collar volume, the average sheet thickness $\delta_{\text {ave }} \sim 2.9 \mu \mathrm{m}$, in the final panel of the figure. This is further segmented by the pulling of folds around the vortex ring, showing that the finest sheets are certainly less than 1 micron thick. Direct imaging can only be used for estimating an upper bound for the localized thickness of these sheets due to diffraction in the side view. The bottom-most section of the viscous sheets in Figs. 13(a) and 13(b) give an estimate of about $\delta<3 \mu \mathrm{m}$.

Not only do the double sheets become thinner during the winding-induced stretching, but their separation distance reduces, as the pool liquid between them is confined and is also stretched. This is clear by observing the tip of the folded sheet in Fig. 10, marked by the arrows, which rapidly becomes thinner as it winds around the vortex. In Sec. III E, we construct a simple model of the stretching, which supports similar nonuniform stretching along the sheets.

Figure 15 shows even more windings around the vortex ring, as it has traveled further into the pool. It is not possible to track all the sheets at this stage, as they have become too thin. Figures 15(b) and 15(c) show other close-up images. Many of the wrappings are double but cannot be separated in the image. Keep in mind that the two liquids are fully miscible and thereby lack surface tension. The sheets will therefore not rupture, only thin and eventually diffuse into the surrounding pool to loose their identity. The Peclét number $\mathrm{Pe}=U \delta / \beta$, where $\beta$ is the molecular diffusivity of the highly viscous drop liquid into the pool, is an indication of the strength of the molecular diffusion vs advection. As $\beta \sim 10^{-11} \mathrm{~m}^{2} / \mathrm{s}$ [25,48], Pe takes a value, based even on the sheet thickness, which is $\gg 1$ and advection dominates over diffusion during the short times ( $\sim 50 \mathrm{~ms})$ involved in our imaging. 


\section{LI, BEILHARZ, AND THORODDSEN}

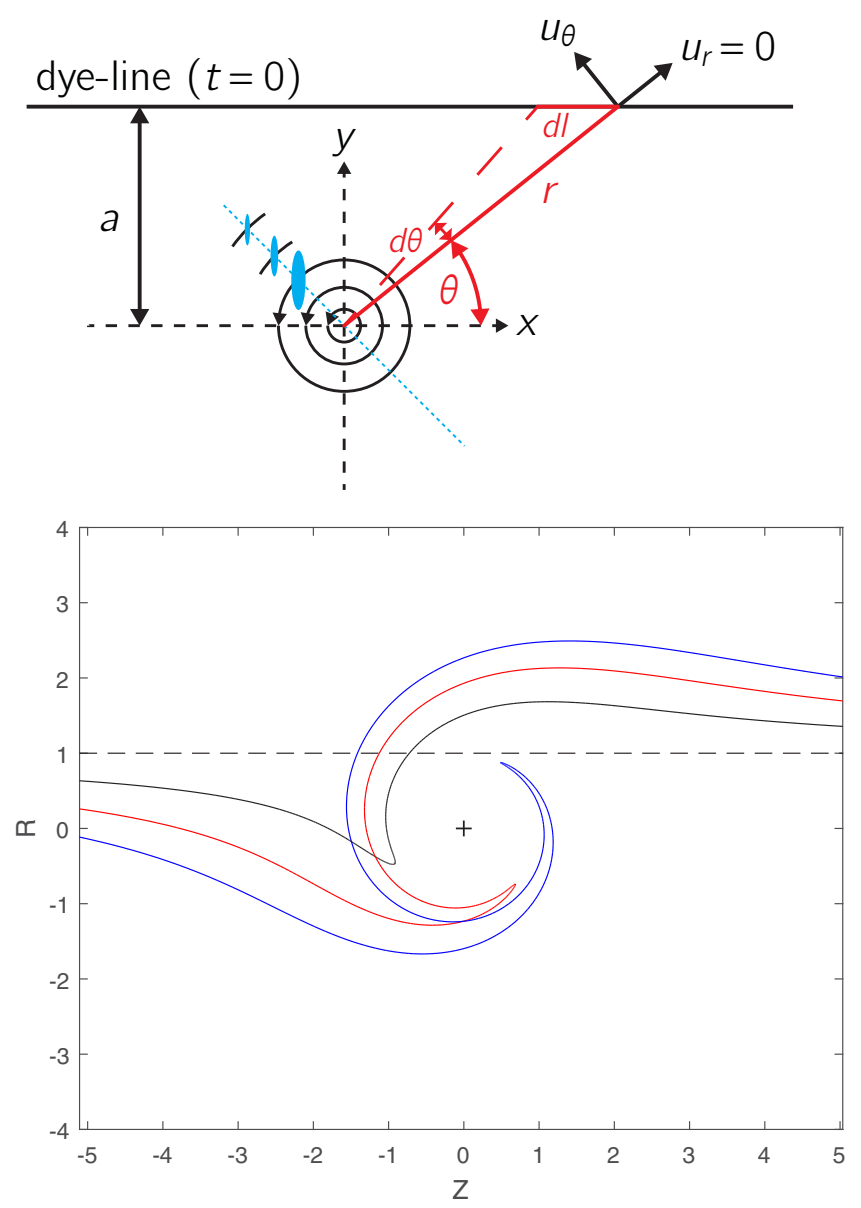

FIG. 18. Top: Schematic setup of the simple model. The ellipses indicate the orientation and strength of the principal rates of strain. Bottom: Numerical results showing the wrapping of a dye line (dashed horizontal line) around a stationary point vortex (+), shown at three different times. The fluid elements along the streak are parametrized by their initial angle $\theta^{*}$ and evolve in time in accordance with Eq. (2). The leading section of the fold becomes thinner, just like in the experiments in Fig. 10.

\section{E. Simple winding model}

The simplest qualitative model of the winding of a viscous sheet by a vortex ring is that of a dyed line advected by a two-dimensional (2D) point vortex of strength $\Gamma$, originally located at a distance $a$, as sketched in Fig. 18(a). We define the center of the vortex as the origin and place a straight dye line $(y=a)$ at $t=0$. The fluid elements along the dye line are parametrized by their initial angle $\theta^{*}$ and their respective distance to the vortex center is $r=a / \sin \theta^{*}$. When time goes on, each fluid element of the dye line remains at its original distance $r$ from the point vortex and simply rotates around it with a constant speed $u_{\theta}=\Gamma /(2 \pi r)=\Gamma \sin \theta^{*} /(2 \pi a)$. The shape of the dye line at time $t$ is therefore

$$
\theta\left(\theta^{*}, t\right)=\theta^{*}+\frac{\Gamma t}{2 \pi a^{2}} \sin ^{2} \theta^{*}, \quad \theta^{*} \in\left[0^{\circ}, 180^{\circ}\right]
$$

Varying the initial angle $\theta^{*}$ from $0^{\circ}$ to $180^{\circ}$ traces out the entire curve at a fixed time $t$. Figure $18(\mathrm{~b})$ shows how a fold is pulled around to form a thinning double-sided finger, the tip of which thins with 
time. Even this simplest model shows the uneven stretching of the sheet. At the start of the motions, the left side of the dye line is in compression, while the right side is in extension. In Cartesian coordinates, along the original line $\mathbf{x}=(x, y=a)$, the horizontal velocity is

$$
u=-\frac{\Gamma}{2 \pi r} \sin \theta^{*}=-\frac{\Gamma}{2 \pi} \frac{a}{\left(x^{2}+a^{2}\right)} .
$$

with the corresponding horizontal strain-rate along the dye line of

$$
e_{x x}=\frac{\partial u}{\partial x}=\frac{\Gamma}{\pi} \frac{x a}{\left(x^{2}+a^{2}\right)^{2}} .
$$

This is antisymmetric about $\theta=90^{\circ}$ showing a maximum and minimum at $x= \pm a / \sqrt{3}$, where $\theta^{*}=60^{\circ}$ and $120^{\circ}$. To find the actual stretching rate, we need to consider the full strain-rate tensor. For a point vortex, the direction of the principal rate of strain is aligned at $45^{\circ}$ away from the radial direction, but its magnitude decreases $\propto 1 / r^{2}$, with distance away from its center. See ellipses in Fig. 18(a). By projecting the line element $\mathbf{d} \ell$, along $y=a$, onto the local directions of the principal rates of strain, we can estimate the full local strain rates as

$$
\left|\mathbf{e}_{i j} \cdot \mathbf{d} \ell\right|=\frac{\Gamma}{r^{2}}\left[\cos \left(\theta^{*}-\pi / 4\right)+\cos \left(\theta^{*}+\pi / 4\right)\right],
$$

which simplifies to $\left(\sqrt{2} \Gamma / a^{2}\right) \sin ^{2} \theta^{*} \cos \theta^{*}$, which is antisymmetric around $\theta^{*}=90^{\circ}$. The maximum is at $\theta^{*}=\arctan \sqrt{2} \approx 54.7^{\circ}$ and the minimum at $\theta^{*}=\pi-\arctan \sqrt{2} \approx 125.3^{\circ}$, with peak extension/compression rates of $\pm 2 \sqrt{2} \Gamma /\left(3 \sqrt{3} a^{2}\right)$. The antisymmetry is quickly broken as the streak starts advecting to produce total stretching on both sides.

We can determine the stretching of the dye line by considering the evolution of a distance $d l$ between two fluid elements originally located at $\theta^{*}$ and $\theta^{*}+d \theta^{*}$ as sketched in Fig. 18(a):

$$
\begin{aligned}
d l\left(\theta^{*}, t\right)= & \left(\left[\frac{a}{\sin \left(\theta^{*}+d \theta^{*}\right)} \sin (\theta+d \theta)-\frac{a}{\sin \theta^{*}} \sin \theta\right]^{2}\right. \\
& \left.+\left[\frac{a}{\sin \left(\theta^{*}+d \theta^{*}\right)} \cos (\theta+d \theta)-\frac{a}{\sin \theta^{*}} \cos \theta\right]^{2}\right)^{\frac{1}{2}} .
\end{aligned}
$$

Inserting Eq. (2) and neglecting second-order terms yields

$$
d l\left(\theta^{*}, t\right) \approx \frac{a d \theta^{*}}{\sin ^{2} \theta^{*}} \sqrt{\left(1+\frac{\Gamma t}{2 \pi a^{2}} \sin 2 \theta^{*}\right)^{2} \sin ^{2} \theta^{*}+\cos ^{2} \theta^{*},}
$$

where the term in front of the square root can be identified as $d l\left(\theta^{*}, t=0\right)$. Hence the total stretch of the fluid elements along the sheet is

$$
\frac{d l\left(\theta^{*}, t\right)}{d l\left(\theta^{*}, t=0\right)} \approx \sqrt{\left(1+\frac{\Gamma t}{2 \pi a^{2}} \sin 2 \theta^{*}\right)^{2} \sin ^{2} \theta^{*}+\cos ^{2} \theta^{*} .}
$$

Figure 19(b) depicts the temporal evolution of this total strain of the fluid elements along the sheet as a function of their original location in $x$. The total stretching now occurs over the entire sheet, except near the central point where the fold occurs, indicated by values less than 1 . There remains a region of total compression near the tip of the finger which leads the wrapping of the sheet. The location of this tip is defined as

$$
\frac{\partial \theta}{\partial \theta^{*}}=1+\frac{\Gamma t}{2 \pi a^{2}} \sin \left(2 \theta^{*}\right)=0
$$



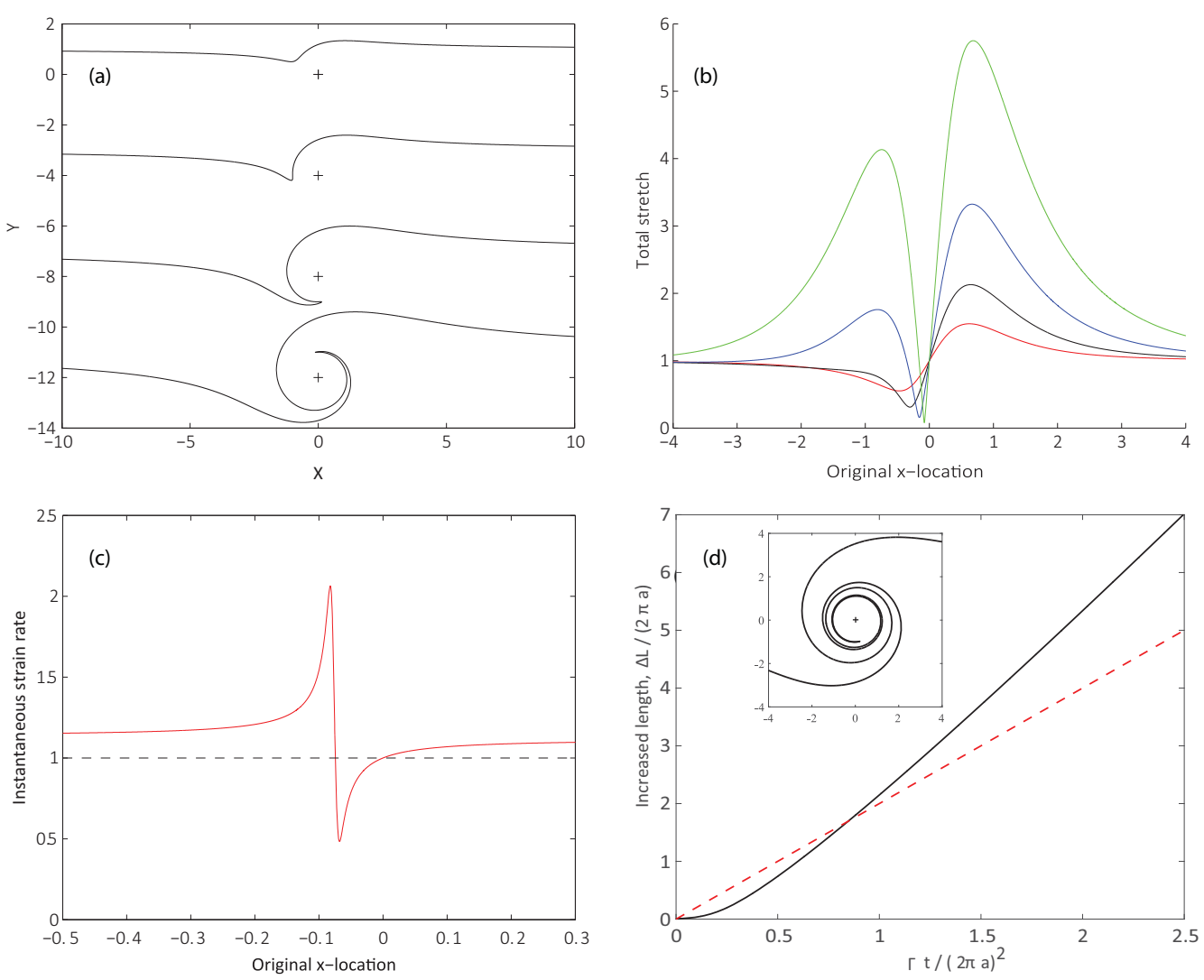

FIG. 19. Line stretching from the simple vortex model. (a) The deformation of the dye line shown at $\Gamma t=5$, 10,20 , and 40. The minimum distance of the line from the vortex is $a=1$. (+) marks the vortex location. Subsequent shapes have been shifted down to minimize clutter. (b) The corresponding total stretch with time (red, black, blue, green), plotted against the original location of the fluid element in the $x$ direction. (c) The instantaneous strain rate (arbitrary units) along the sheet at $\Gamma t=40$. Note the greatly reduced horizontal extent of this plot compared to the previous panel. (d) The total lengthening of the dye line $\Delta L$ vs time (solid line). The dashed line has a slope of 2, representing the simple model of Eq. (3). The inset shows the shape of the dye line at $t / T_{a}=2.5$.

and it first forms for the fluid element originally at $\theta^{*}=135^{\circ}$ at time $t=2 \pi a^{2} / \Gamma$. Perhaps counterintuitively, the tip of the finger does not remain at the same radial distance from the point vortex. As $t$ increases, $\sin \left(2 \theta^{*}\right)$ approaches zero or $\theta^{*} \Rightarrow 90^{\circ}$, where the radius at the tip goes to the minimum possible value, i.e., $r=a$. This can be understood in the following manner: At the tip, the slope of the sheet is tangent to a radial ray from the center of the point vortex. At that leading point, there is no velocity component along the sheet. Whereas along the sheet below this point, i.e., for slightly smaller $r, u_{\theta}$ is larger, pushing the sheet toward the fingertip, thereby locally compressing it. This we show by plotting the instantaneous strain rate in Fig. 19(c), as opposed to the total strain in Fig. 19(b). Keep in mind that the tip of the fold slowly shifts toward $r=a$ and is therefore not composed of the same fluid elements. The width of the fingertip, i.e., the distance between the two sheets, becomes thinner. Since no fluid element moves in the radial direction, this also implies redistribution of these fluid elements along the sheet.

Thickening of the fluid below the tip is not obvious in the experiments, but some indications are present in the two tips in Figs. 12(b) and 12(c). 


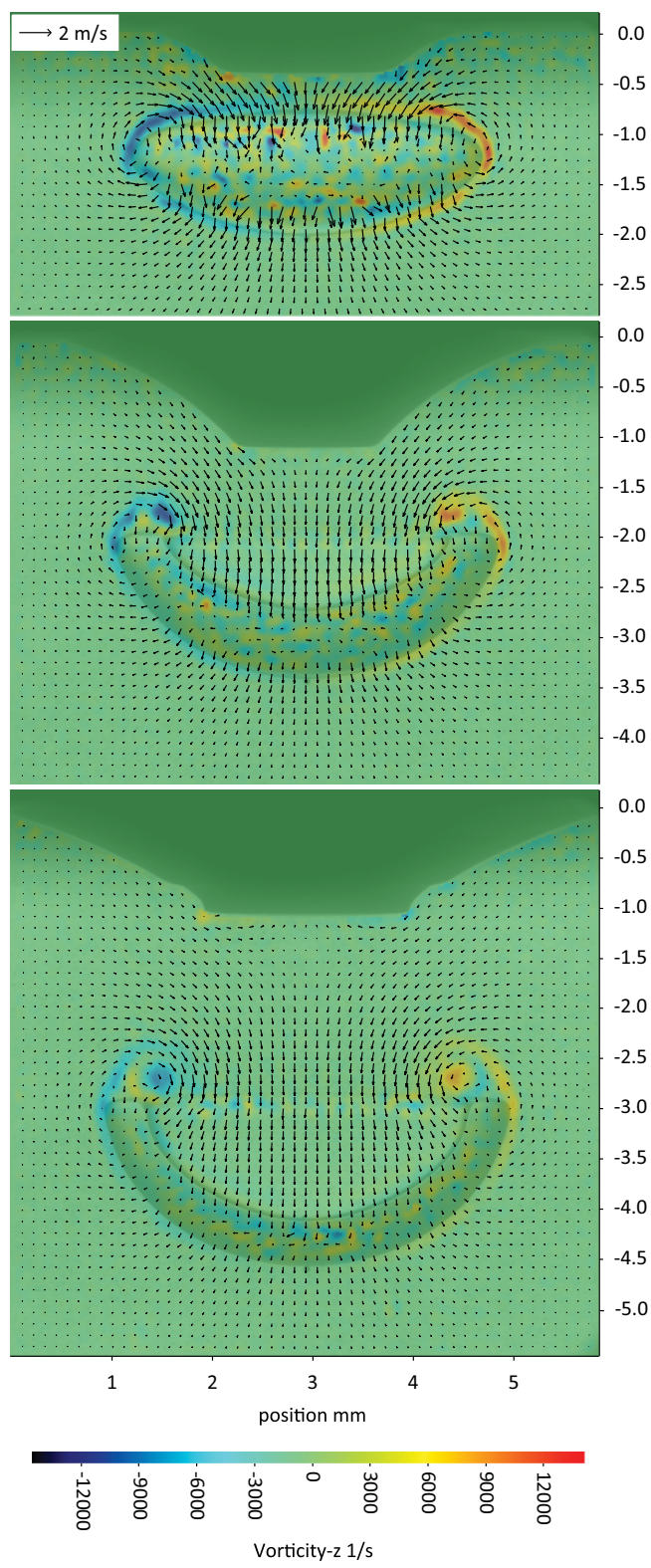

FIG. 20. The formation of the vortex ring, for $H=180 \mathrm{~mm}, U=1.65 \mathrm{~m} / \mathrm{s}, v_{d}=500 \mathrm{cSt}, v_{p}=1 \mathrm{cSt}$ $\left(\operatorname{Re}_{p}=3993, \mathrm{We}_{p}=319\right)$. Top panel: the boundary layer separates around the equator of the drop. Middle and bottom panels: The separated vortex sheet starts to roll up into a vortex ring. Frames shown at $t=1.9,3.7$, and $6.5 \mathrm{~ms}$ after first contact. The color indicates the strength of the azimuthal vorticity.

Figure 19(d) shows the total increase in the length of the dye line $\Delta L$ vs time. We normalize the time by the rotational period of a fluid element at $r=a$, i.e., $T_{a}=(2 \pi a)^{2} / \Gamma$, while $\Delta L$ is normalized by the periphery of the circle with $r=a$. The evolution of $\Delta L$ is qualitatively similar to the growth of the drop area, from the experiments shown in Fig. 16(c). It shows slow initial increase, followed by a faster growth at a constant rate. The dashed line in the figure represents the increase in $\Delta L$ owing to a simple model, where a double-walled sheet, like that in Fig. 10, is pulled around the 

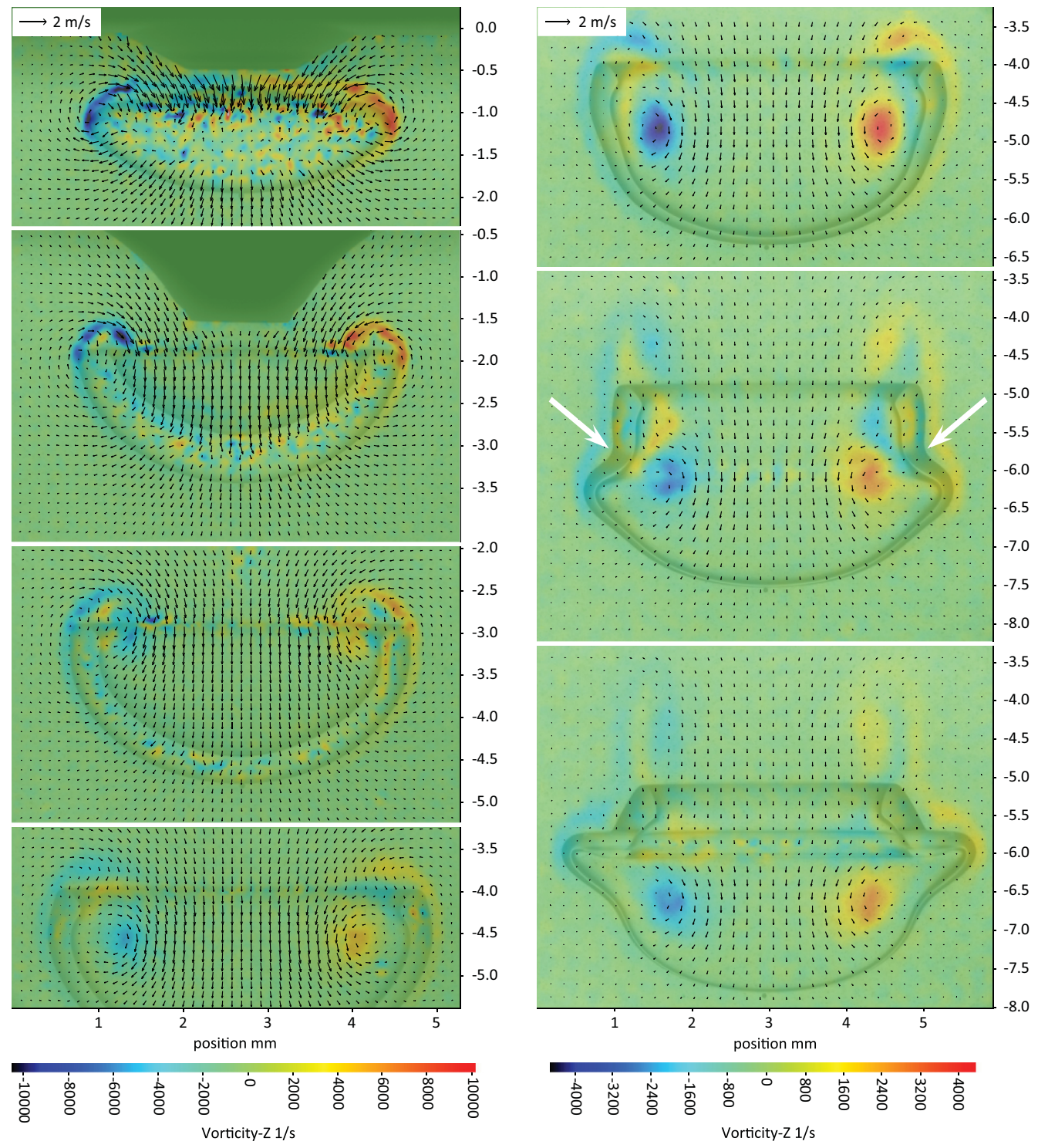

FIG. 21. The vortex ring formation and advection into the bowl, for $H=240 \mathrm{~mm}, U=1.93 \mathrm{~m} / \mathrm{s}, v_{d}=$ $500 \mathrm{cSt}, v_{p}=1 \mathrm{cSt}\left(\operatorname{Re}_{p}=4671, \mathrm{We}_{p}=436\right)$. The separated vortex sheet rolls up into a vortex ring and enters the bowl-shaped drop. The induced motion generates opposite sign vorticity from the inner edge of the drop. Frames shown at $t=1.6,3.4,6.2,10.5,10.9,16.4$, and $20 \mathrm{~ms}$ after first contact. Only every 4 th velocity vector is plotted to avoid clutter. The color codes the strength of the azimuthal vorticity. Note that the color scale is different from the left and right columns, with lower maximum values in the right column.

vortex at the speed $u_{\theta}=\Gamma /(2 \pi a)$. This gives $\Delta L=2 u_{\theta} t=\Gamma t /(\pi a)$, which in normalized form becomes

$$
\Delta L /(2 \pi a)=2 t / T_{a} .
$$

$\Delta L$ is shown to grow at a faster rate than this due to stretching of the outermost layers.

In this simple model, only one fold is pulled around the vortex, but one can envision a second-order model where two point vortices are allowed to self-advect with the flow, which may entrain other 


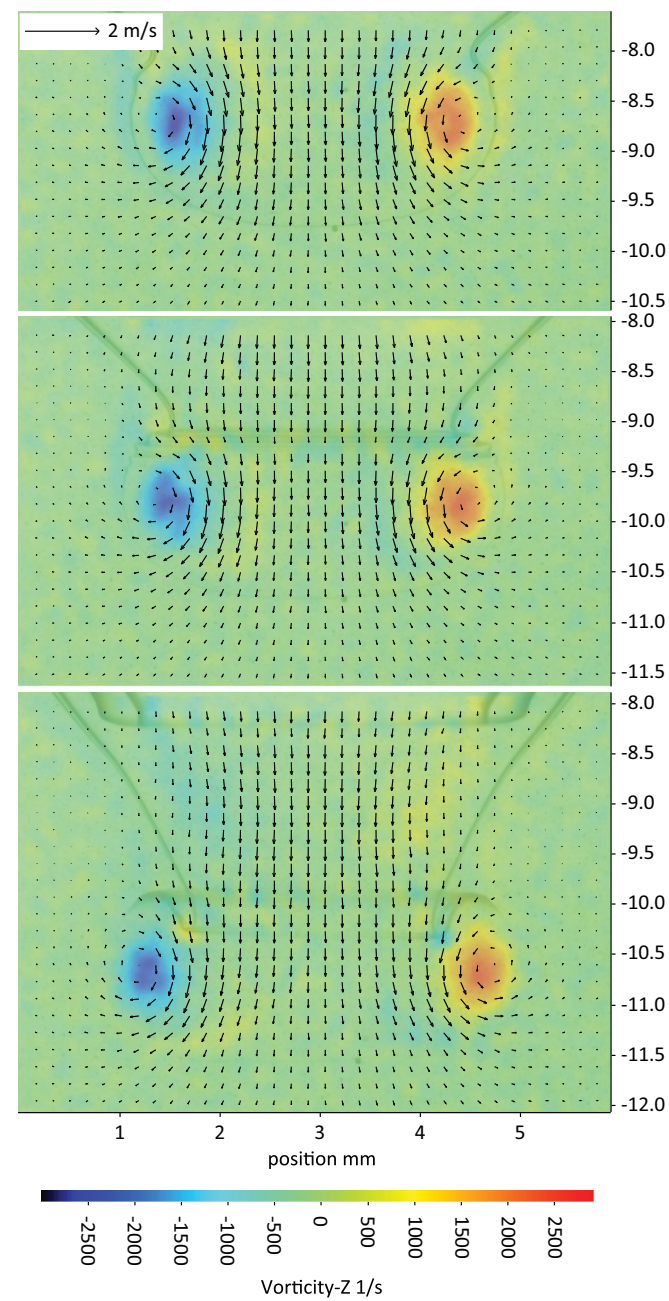

FIG. 22. The motion of the vortex ring inside the bowl, for the same conditions as in Fig. 21. Axisymmetric buckling is visible above the vortex ring in the middle panel. In the bottom panel, the vortical motions start pulling in a fold, as in Fig. 7. Frames shown at $t=29.8$, 35.0, and $38.7 \mathrm{~ms}$ after first contact. The color levels of the azimuthal vorticity are lower than those in Fig. 21.

folds, as is observed in the experiments. Furthermore, the axisymmetry would be needed to reproduce possible azimuthal compression. This is currently being investigated for a future report. However, keep in mind that our sheets are not passive tracers but inject localized viscous stress.

\section{F. PIV results: The vortex ring}

In a set of experiments, the pool liquid was seeded with fine particles to quantify the velocity field and follow the formation and evolution of the vortex ring. The most important issue is here the origin and strength of the vorticity. In homogeneous drop impacts, before first contact the drop and pool contain no vorticity. The principle of persistence of irrotationality therefore demands that the vorticity must be produced in the free surface, by flow along a curved interface ([49], pp. 276-277). See, for example, the discussion in Brøns et al. [50] and Thoraval et al. [37,51]. For the same low-viscosity liquid in the drop and pool, the vortex ring is produced by separation of 

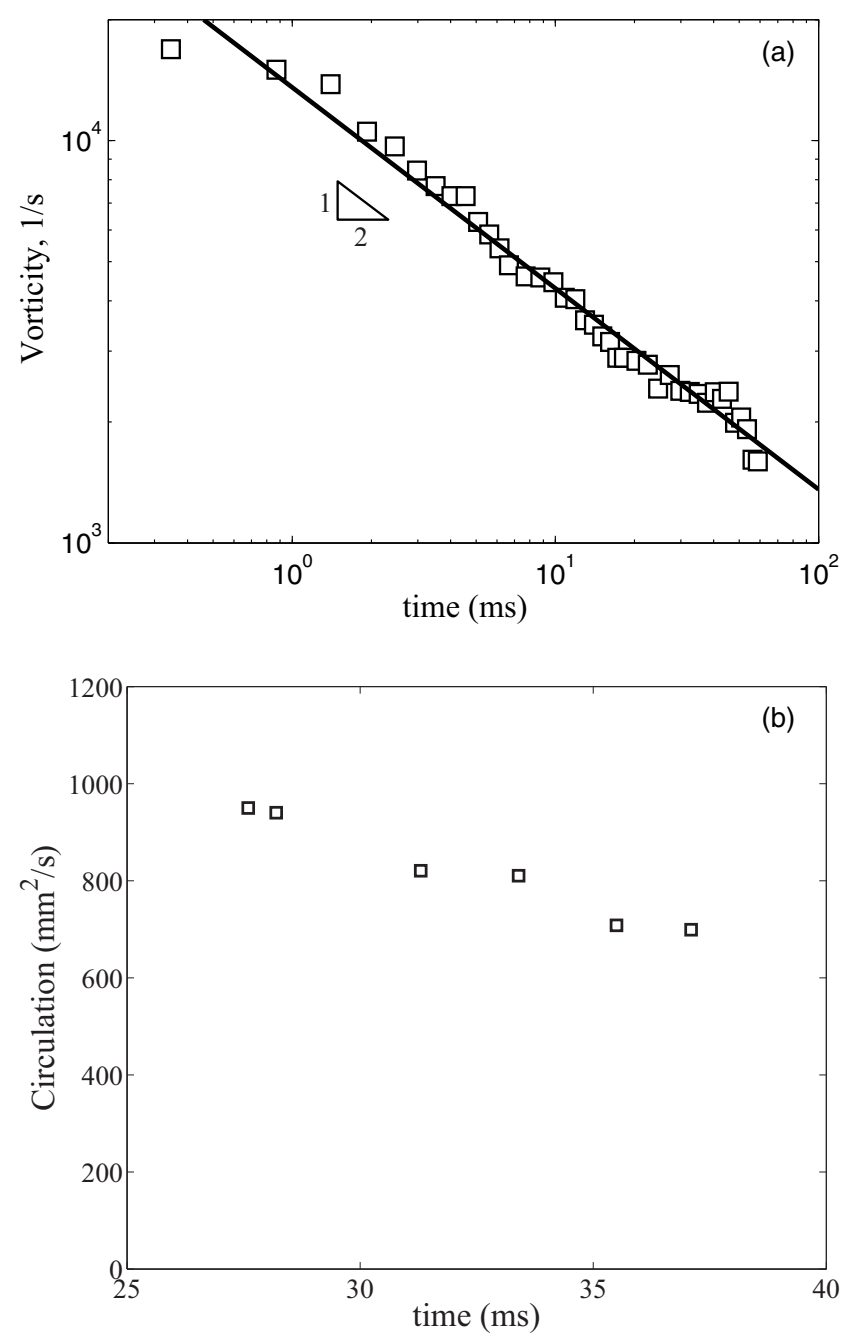

FIG. 23. (a) The maximum vorticity in the core of the vortex ring, as it travels down into the pool. The line has a slope of $-1 / 2$. Constructed from a few identical realizations taken at the same magnifications but at different depths within the pool, for $U=1.93 \mathrm{~m} / \mathrm{s}, \mathrm{Re}=4671$; We $=436$. (b) Circulation of the vortex ring after it has rolled up and entered the bowl, for the same conditions as in (a).

a vortical boundary layer in the neck connecting the drop and the pool, during the early stage of the impact [36]. The motion of the neck is in turn driven by capillary-inertial dynamics, which require low Weber number impacts, where a critical $\mathrm{We}_{c}<64$ has been suggested by Hsiao et al. [34]. Herein, we both have We $\gg \mathrm{We}_{c}$, but more importantly, the large viscosity of the drop excludes the above mechanism for the vortex generation. On the other hand, in the present configuration, the liquid domain is not homogeneous, differing greatly in viscosity and to lesser extent in density. Density variations can generate vorticity through the baroclinic term in the vorticity equation, $D \omega / D t=-(\nabla \rho \times \nabla p) / \rho_{o}^{2}$, where $p$ is the hydrodynamic pressure. However, this term is not the source of our vortex ring. The PIV results show clearly where the vorticity originates, i.e., in the flow up along the underside of the viscous drop liquid. Figure 20 shows the velocity vectors, along with the azimuthal vorticity field (out of the plane), the intensity of which is marked by the background color. The background includes shading from the first image of the image pair used in the PIV, 


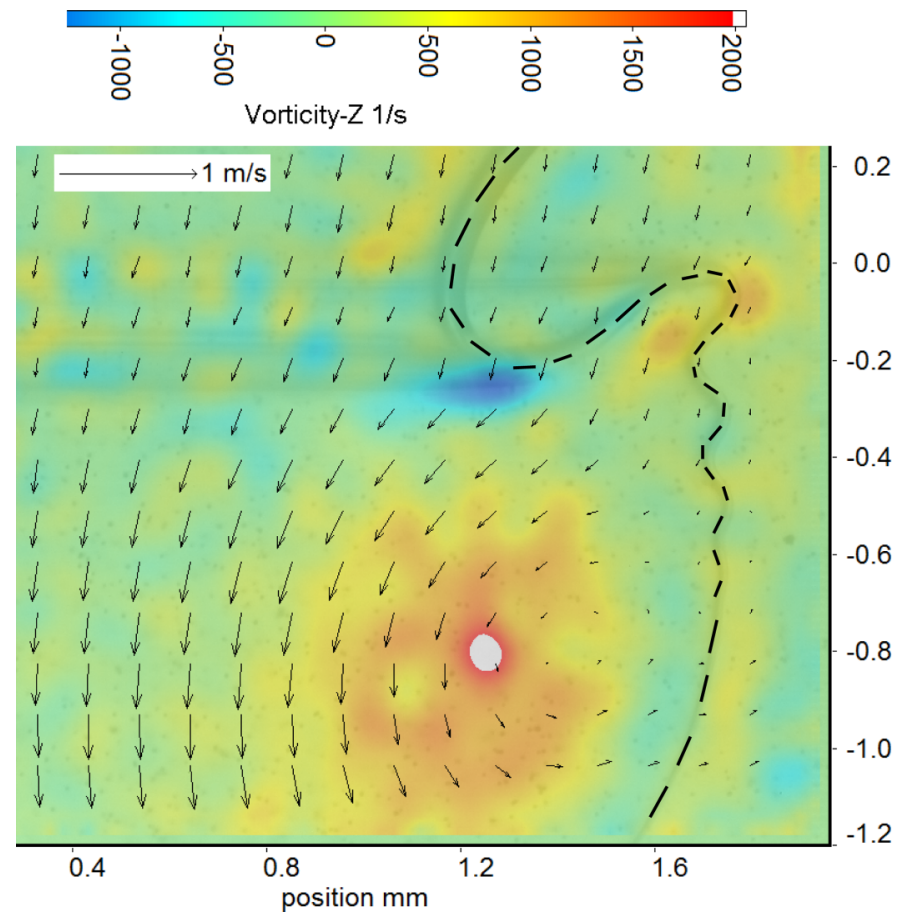

FIG. 24. The velocity vectors around the compressively buckling sheet, for conditions similar to those shown in Figs. 7 and 20, i.e., $U=1.65 \mathrm{~m} / \mathrm{s}, \operatorname{Re}_{p}=3993, \mathrm{We}_{p}=319$. The background color indicates the strength of the azimuthal vorticity. The viscous sheet has been highlighted with a dashed curve, drawn from the background shaded image.

showing the outline of the drop and the deformed pool surface. The figure shows that the vorticity outside this boundary layer is near zero, excluding small localized vorticity in the corner of the crater in the free surface and which is of opposite sign to the vortex ring.

Inertia from the drop impact and the slightly larger drop density pull the drop downward and deform it into a bowl shape, as shown in earlier sections. The pool liquid must therefore flow up along the sides of this descending bowl. The much larger drop viscosity $\left(\mu_{d} / \mu_{p} \simeq 500\right)$ effectively acts like a partial-slip solid surface, through the matched shear stress, thus generating vorticity. This vortical layer is shed from the sharp corner at the top of the bowl and rolls up into the vortex ring. The induced motion around the vortex generates weaker vorticity of opposite sign along the inner corner of the drop (Figs. 20-22); see, for example, the yellow patch inside the blue vortex sheet on the left side of the middle panel in Fig. 20. This vorticity is pulled up into the vortex ring, reducing its strength. In Fig. 23, we track the strength of the vortex ring from its formation.

Figure 21 shows the vortex ring after it has entered the bowl. The boundary layer along the outer layer of the drop continues to separate from the widest section of the bowl and now rises well above the rim, before taking a sharp U-turn back toward the drop. Keep in mind that strong optical refraction at the top of the bowl and at the large folds reduce erroneously the magnitude of the velocity vectors and produce noise in the vorticity field. However, this does not significantly distort the measured vorticity after the vortex has entered into the bowl. The overall rotational flow contributes, along with the buoyancy, to the large-scale buckling of the rather thick $(\sim 0.25 \mathrm{~mm})$ drop liquid in the upper section of the hemispheric bowl; see arrows in the second right panel of Fig. 21. Strong opposite-sign vorticity layers are now produced along the inner side of the viscous sheets by the rotational velocity around the vortex core. This vortical layer becomes less prominent 

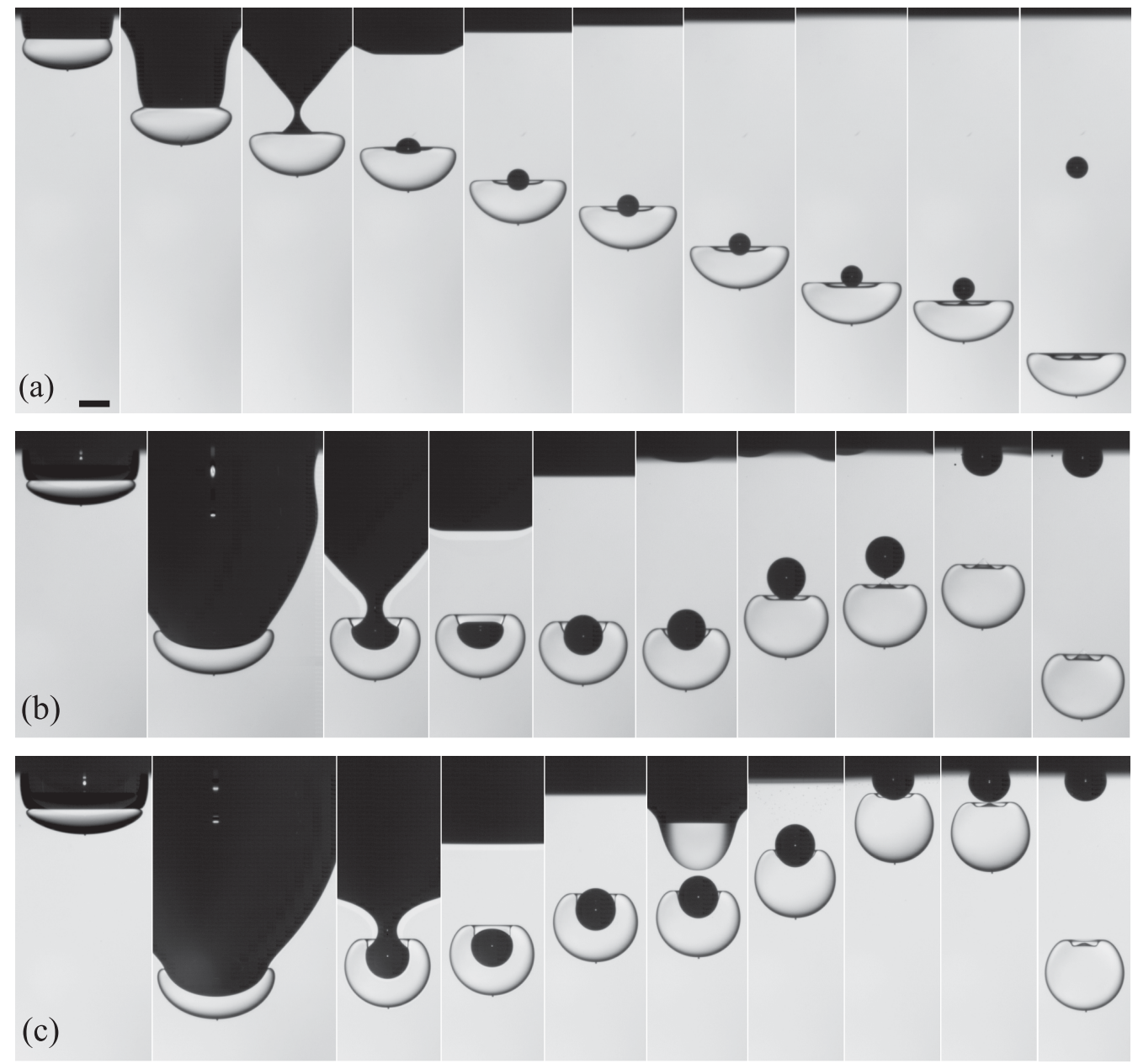

FIG. 25. Effect of increasing the pool viscosity tenfold, shown for three impact velocities. Impact of 500-cSt drop onto 10-cSt pool. (a) $U=1.12 \mathrm{~m} / \mathrm{s}\left(\operatorname{Re}_{p}=275 ; \mathrm{We}_{p}=145\right)$. The panels are shown at $t=13,46,77$, 98, 162, 241, 439, 733, 950, and $1880 \mathrm{~ms}$. (b) $U=2.08 \mathrm{~m} / \mathrm{s}\left(\operatorname{Re}_{p}=504 ; \mathrm{We}_{p}=495\right)$, at $t=1.3,15,23,25$, 48, 136, 251, 276, 422, and $1000 \mathrm{~ms}$. (c) $U=2.47 \mathrm{~m} / \mathrm{s}\left(\operatorname{Re}_{p}=599 ; \mathrm{We}_{p}=698\right)$, at $t=1.2,18,26,29,69$, $134,209,295,716$, and $1520 \mathrm{~ms}$. The bump in the sixth panel is a satellite drop, likely pinched off from the Worthington jet, supported by a thin Mesler air film [40,44], which does not change the overall dynamics. The scale bar is $1 \mathrm{~mm}$ long. See also videos in the Supplemental Material [43].

in Fig. 22, where the viscous sheet is much thinner and therefore provides less viscous stress but is rather advected and stretched by the flow field.

Eventually, the vortex ring travels down and reaches the bottom of the growing bowl, driven by mutual induction and starts to wrap this sheet around, as was shown in an earlier subsection. At this stage, the viscous sheets of drop liquid are quite thin and may only have a secondary effect on the dynamics.

Figure 24 shows a close-up view of how the vortex ring causes compressive stress along the viscous sheet. In the laboratory frame, the outer edge of the vortex slows the vertical velocity to zero, while the upper part of the sheet is moving downward. In this particular case, the downward tangential velocity at the location of the viscous sheet reduced from about $0.14 \mathrm{~m} / \mathrm{s}$ to zero, over a distance of $0.7 \mathrm{~mm}$, giving $\partial w / \partial z \simeq-200 \mathrm{~s}^{-1}$, as was already determined from the frame sequences 

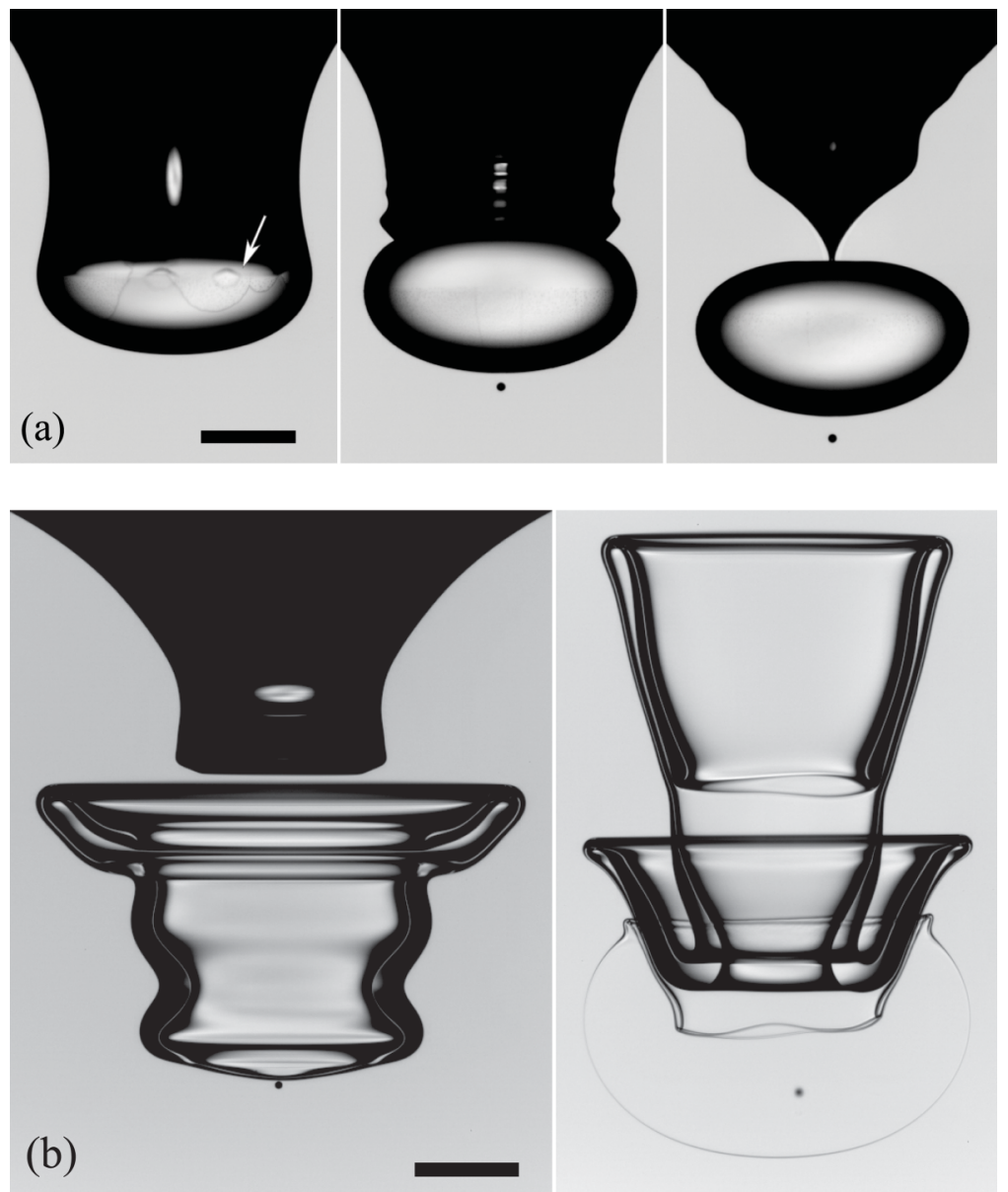

FIG. 26. Impact of a 100-cSt drop onto a pool of $0.65 \mathrm{cSt}$. (a) The breakup of the Mesler film under the drop, for $U=0.69 \mathrm{~m} / \mathrm{s}, \operatorname{Re}_{p}=2569, \mathrm{We}_{p}=55$. The arrow points at a line of ruptures of the air film, which subsequently contracts into a bottom bubble [44]. (b) Buckled shapes for $U=1.17 \mathrm{~m} / \mathrm{s}, \operatorname{Re}_{p}=4356, \mathrm{We}_{p}=$ 158. The "bull-shape" on the left side emerges at $21 \mathrm{~ms}$ and the multiple folds at $53 \mathrm{~ms}$ after impact. The panels are at different depths. The scale bars are $1 \mathrm{~mm}$ long.

in Fig. 9. We also note that the vorticity next to the sheet is much weaker than in the vortex core but changes sign across the sheet, suggesting boundary layers with the flow moving up on both sides, relative to the tangential motion of the sheet and thereby compressing it.

Figure 23(a) shows the time evolution of the maximum azimuthal vorticity in the vortex core. As expected the magnitude of $\omega_{\max }$ falls off as $\sim t^{-1 / 2}$, characteristic of viscous diffusion. Owing to our fine spatial resolution, we can accurately measure this maximum magnitude, which reduces from $16000 \mathrm{~s}^{-1}$ by a factor of 10 to $\sim 1600 \mathrm{~s}^{-1}$ in $60 \mathrm{~ms}$, which corresponds to about $\tau=t R / U=87$ in normalized impact time units. The oscillations in the signal are real and arise from the interactions of the vortex ring with the viscous surface of the drop liquid. This maximum vorticity will only be slightly under-resolved during the earliest times, as the characteristic viscous scale in the pool boundary layer, along the bottom side of the drop, is quite thin. If we estimate this thickness within the pool as $\delta \simeq \sqrt{v_{p} t}$, over the first ms, we get $\delta \simeq 32 \mu \mathrm{m}$, whereas the smallest interrogation window used in the multistep cross-correlation procedure, in Fig. 20, is $16 \times 16$ px corresponding to $32 \mu \mathrm{m}$ The close-up imaging in Fig. 24 has finer spatial resolution of $12 \times 12 \mathrm{px}$ of $24 \mu \mathrm{m}$. Keep in mind that only every fourth vector is plotted in the figures. Even though the peak core 


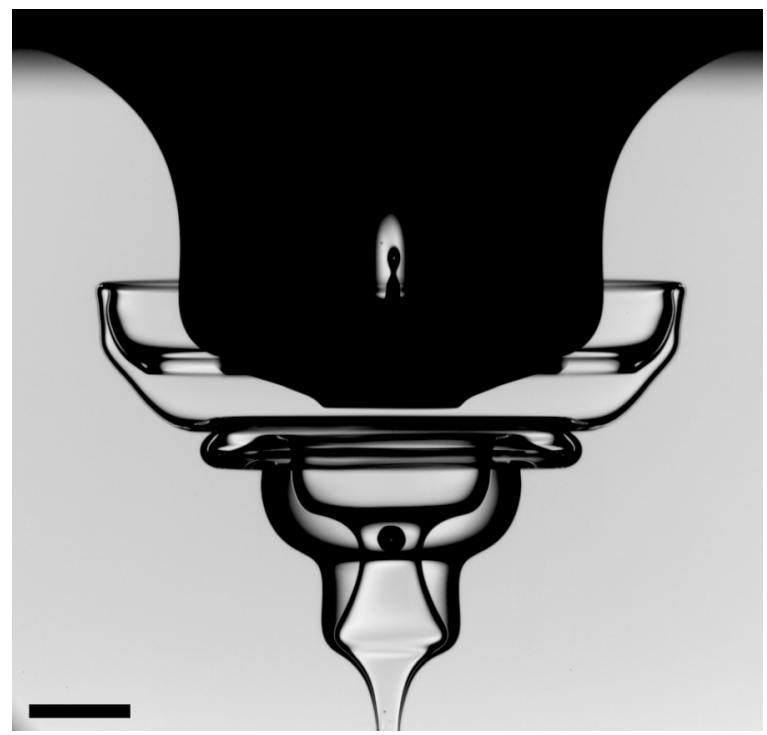

FIG. 27. Close-up of the shape for 100-cSt drop impacting 0.65 -cSt pool, for $U=1.45 \mathrm{~m} / \mathrm{s}, \operatorname{Re}_{p}=5398$, $\mathrm{We}_{p}=243$, shown at $t=20 \mathrm{~ms}$. Notice the jet inside the crater and one bubble entrapped on the centerline. The scale bar is $1 \mathrm{~mm}$.

vorticity reduces rapidly, the circulation remains nearly constant, reducing only by $25 \%$, as plotted in Fig. 23(b). The circulation is not fully conserved here, as it entrains some of the boundary layer formed at the inner edge of the viscous sheet, as was shown in Fig. 21.

PIV becomes difficult as the vortex travels further into the pool and wraps the viscous sheets repeatedly. We can, however, roughly estimate the circulation from the speed of the wrapping of the sheets. Figure 15 showed the vortex ring further down inside the pool. We can estimate the circulation by the wrapping of the innermost viscous layer around the vortex core, giving the rotation velocity of the tip, $u_{\theta}=0.41 \mathrm{~m} / \mathrm{s}$ and $\Gamma=u_{\theta} * \pi L=870 \mathrm{~mm}^{2} / \mathrm{s}$, where $L$ is the diameter of the wrapping. This is in good agreement with the PIV results. In this figure, the vortex ring diameter is $4.1 \mathrm{~mm}$ and it advects downstream at $V=0.113 \mathrm{~m} / \mathrm{s}$.

Figure 15(c) shows a vortex ring even further down, with many irregular buckled sheets visible. Here, the ring diameter is $3.97 \mathrm{~mm}$ and $V=0.118 \mathrm{~m} / \mathrm{s}$, with the corresponding $u_{\theta} \simeq 0.21 \mathrm{~m} / \mathrm{s}$ and $\Gamma=u_{\theta} \pi L=330 \mathrm{~mm}^{2} / \mathrm{s}$. This estimate is lower but of the same order of magnitude as those calculated above from the actual velocity field.

\section{CHANGING THE DROP-POOL VISCOSITY RATIO}

In the previous section, we have kept the drop viscosity at $500 \mathrm{cSt}$ while keeping the pool viscosity quite low, on the order of 1 . In this section, we first investigate how the dynamics are affected if the pool viscosity is increased significantly. Second, we document the convoluted shapes which occur when the drop viscosity is lowered by a factor of 5 to $100 \mathrm{cSt}$.

\section{A. Higher pool viscosity}

When the pool viscosity is increased by a factor of 10 to $10 \mathrm{cSt}$, we do not observe a concentrated vortex ring. Instead, the dynamics are controlled by a bubble which is entrapped by the outer liquid on top of the drop, as the crater closes up above the drop and the pool liquid does not fully wet the drop surface. This is shown in Fig. 25 and occurs over a wide range of impact velocities. This bubble is therefore in direct contact with the drop liquid, partly imbedded into its top surface. Its buoyancy 
pulls the drop upward, against its weight, until the bubble detaches. In Fig. 25(a), the bubble is too small to slow the drop down significantly, whereas in Figs. 25(b) and 25(c) the bubbles are large enough to reverse the motion of the drop-bubble composite. In Fig. 25(c), it can even pull the drop all the way back up to the pool surface. The volume of the bubble in both cases is very similar, but for the higher impact velocity it is embedded slightly further into the drop and takes longer to dislodge itself. Keep in mind that the liquids are miscible, so there is no triple line where they meet at the surface of the bubble [52].

For the bubble to detach from the drop, the pool liquid must intrude between them, as the drop viscosity is too high for the upward buoyancy to rapidly deform it. This will, of course, depend on the viscosity ratio, as well as on the bubble surface tension. The time scale of this intrusion $T_{p}$ is set by a balance between the suction pressure from the bubble buoyancy $\rho_{\text {pool }} g D_{b}^{3}$ and the viscous stress acting against the suction pressure in the film under the bubble $\mu_{\text {pool }} U_{\text {film }} /\left(\delta_{\text {film }}\right)$. Here the film velocity is bounded by no slip at the drop and is shear free at the bubble surface, i.e., $U_{\text {film }} \sim \rho_{\text {pool }} g \delta_{\text {film }}^{2} / \mu$, where $\delta_{\text {film }}$ is the thickness of the intruding film. From this, we form a characteristic time $T \sim D_{b} / U_{\text {film }} \sim \mu D_{b} /\left(\rho_{\text {pool }} g \delta_{\text {film }}^{2}\right)$ and expect detachment when $\delta_{\text {film becomes }}$ a significant fraction of $D_{b}$, or $T_{p} \sim \mu /\left(\rho_{\text {pool }} g D_{b}\right)$.

In sequence Fig. 25(a), the bubble is $D_{b}=0.69 \mathrm{~mm}$ and the time from pinch-off of the cavity, to its detachment from the drop takes $T_{p}=0.87 \mathrm{~s}$. In comparison, the bubbles in Figs. 25(b) and 25(c) are 1.23 and $1.28 \mathrm{~mm}$ in diameter and detach after $T_{p}=0.25$ and $0.69 \mathrm{~s}$ respectively. This shows that a larger bubble size speeds up the detachment, reducing $T_{p}$, but the embedding distance into the drop also plays a strong role.

It is interesting to note that during the early impact the drop has greatly deformed into a pancake shape sitting at the bottom of the crater, whereas during the collapse of this crater, in Figs. 25(b) and 25(c), the resulting flowfield pulls it back toward a spherical shape, even without surface tension between the two miscible liquids.

\section{B. Lowering the viscosity of the drop to $100 \mathrm{cSt}$}

A few experiments were performed where the drop viscosity was lowered from 500 to $100 \mathrm{cSt}$, to ascertain the influence of drop viscosity on the prevalence of the buckling. The lower drop viscosity should allow the drop to deform and stretch out more easily during the early stages of the impact and produce buckles earlier. This is indeed what we observe, with the emergence of much more convoluted shapes. Below we show some typical cases starting from low to high impact velocities.

Figure 26(a) shows a minimally deformed drop for a low impact velocity, where the Mesler air layer breaks along the equator [44] (see white arrow) and leaves a bottom bubble and a pointy pinch-off of the air crater along the top side of the drop.

For a slightly larger $U$, Fig. 26(b) shows three prominent folds in the drop liquid, in the upper sections of the shape. The liquid sheets at the sides of these folds are nearly linear and angled at about $30 \mathrm{deg}$ to the vertical. The bottom viscous hemispheric sheet is very thin but smooth and without a clear presence of a vortex ring, but this was not verified with PIV. Figure 27 shows a case, with slightly larger $U=1.45 \mathrm{~m} / \mathrm{s}$, where multiple axisymmetric bucklings of the thick drop are observed while the crater is still prominent. An isolated bubble is entrapped at the center.

Figure 28 shows an example, for still higher impact velocity at $U=2.10 \mathrm{~m} / \mathrm{s}$, which produces even more convoluted shapes than seen for the higher viscosity drops. Keep in mind that these convoluted features are repeatable, but some of the wrappings occur too close to the free surface and are too obscured by diffraction for us to figure out the detailed interface structures. Satellites generated during the pinch-off from the nozzle may also play some role, in the details along the centerline, but this could not be ascertained.

Here the drop liquid is greatly stretched to span the entire surface of the large crater. These drop liquid sheets near the free surface undergo large distortion and foldings, when the crater starts to rebound and becomes narrower near its bottom. This pulls the drop sheets toward the centerline, compressing and folding the sheets. The white arrow in Fig. 28 points out a cusp in the surface, 

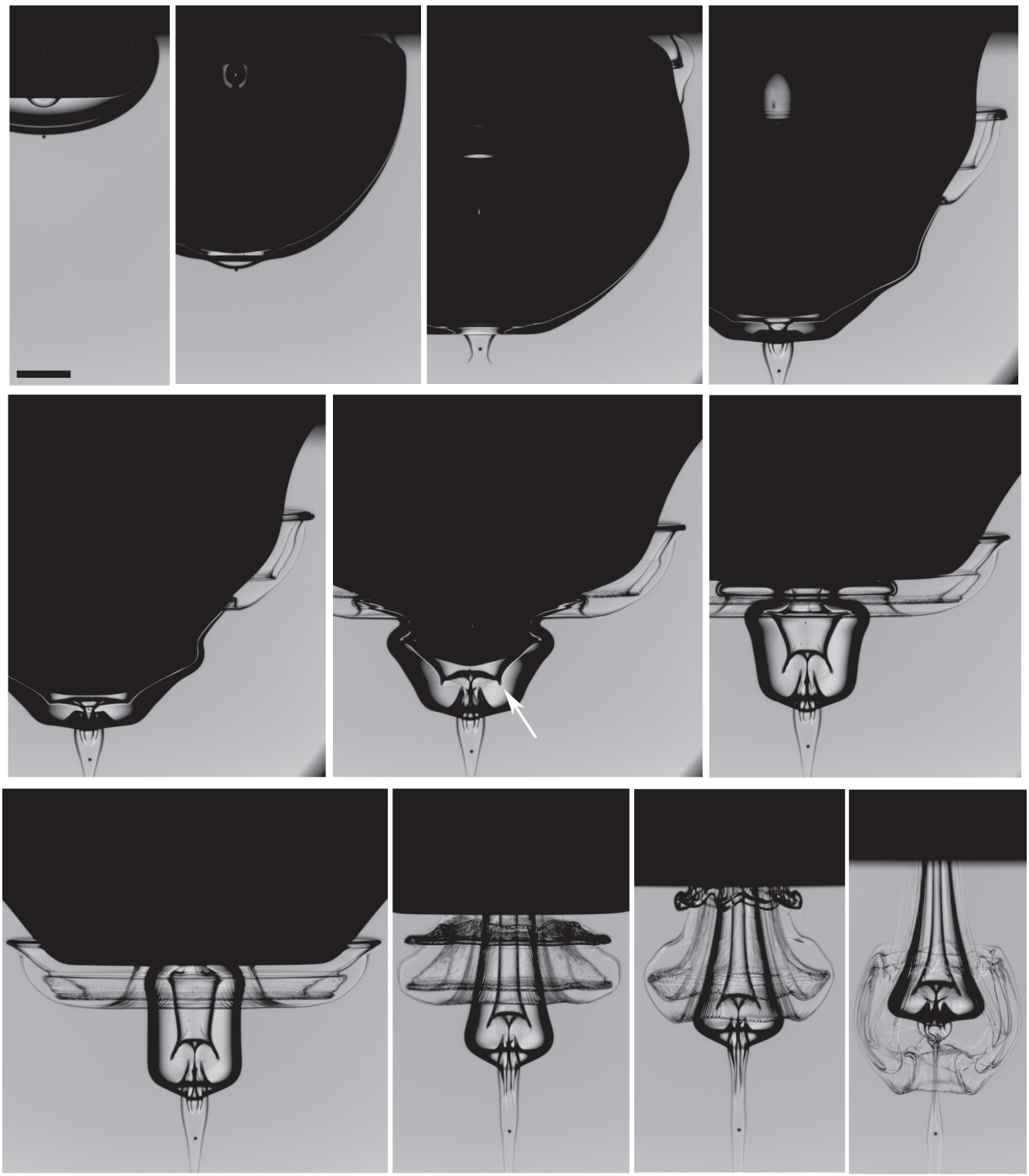

FIG. 28. Time sequence of the complex wrapping of the drop liquid, for a $D=2.42 \mathrm{~mm}$ drop of $v_{d}=100$ cSt impacting onto a 0.65 -cSt pool, for $U=2.10 \mathrm{~m} / \mathrm{s},\left(\operatorname{Re}_{p}=7831 ; \mathrm{We}_{p}=511 ; \operatorname{Re}_{d}=51 ; \mathrm{We}_{d}=494\right)$. Frames shown at $t=1.9,5.7,12.4,21.5,23.0,24.9,26.3,27.8,34.0,39.6$, and $66.8 \mathrm{~ms}$ after impact. Scale bar is $1 \mathrm{~mm}$ long. See also video in the Supplemental Material [43].

which develops as the wave on the crater wall travels down along the free surface, but is damped by the high viscosity drop liquid, which follows the inside of the crater. However, the crater wall is lined with the pool liquid and it enters the viscous cylinder along the axis of symmetry.

The bottom row in Fig. 28 shows fine azimuthal buckles, which are more visible in the close-up images in Fig. 29. The wavelengths are here slightly smaller than for the 500-cSt drop; see, for example, Fig. 9(b). The three regular azimuthal buckles pointed out by the arrows in Fig. 29(a), have original wavelengths of $\lambda \simeq 90 \mu \mathrm{m}$. It is not clear whether this reduced wavelength is due to the lower $v_{d}$, or thinner viscous sheets. This will require more detailed studies, perhaps using viewing 

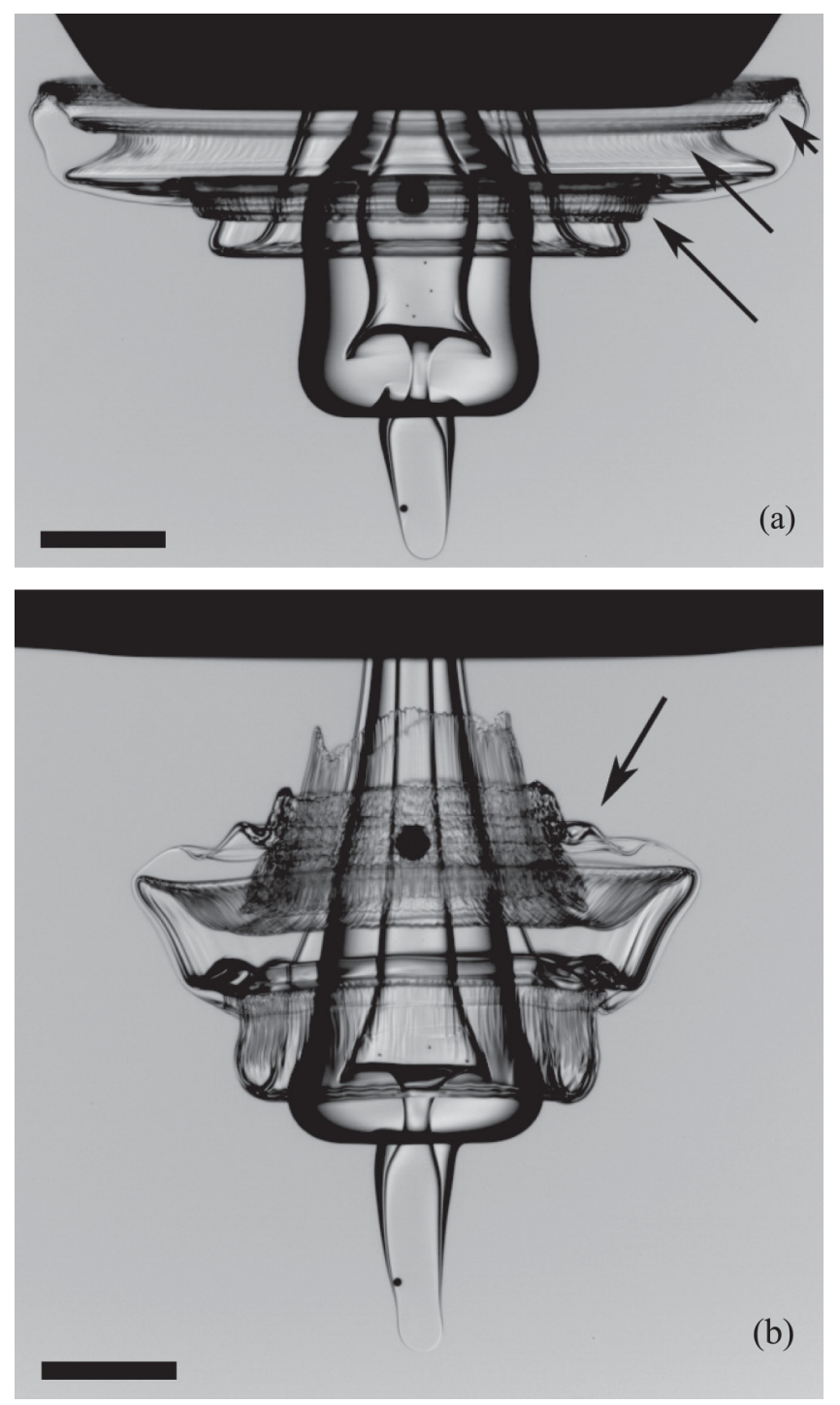

FIG. 29. Complex wrapping of a 100-cSt drop impacting onto 0.65 -cSt pool, for $U=1.90 \mathrm{~m} / \mathrm{s}\left(\operatorname{Re}_{p}=\right.$ 7090; $\mathrm{We}_{p}=418 ; \mathrm{Re}_{d}=46 ; \mathrm{We}_{d}=404$ ). Frame at $t=26.2 \mathrm{~ms}$ (a) and $41.3 \mathrm{~ms}$ (b) after impact. Top image shows three layers with azimuthal buckles. The scale bars are $1 \mathrm{~mm}$ long. See also video in the Supplemental Material [43].

from the bottom. The bottom panel of this figure also shows radial buckles (arrow). Figure 30 shows azimuthal bucklings at the top of the drop liquid, for both high and low drop viscosities.

\section{DISCUSSION AND CONCLUSIONS}

Herein we have identified complex liquid buckling which occurs during the impact of a highly viscous drop on a less viscous but miscible pool. The drop is of slightly larger density than the pool, making it sink. The drop liquid is stretched out into thin viscous sheets, which are susceptible to compression-induced buckling. Both axisymmetric and azimuthal buckling is observed and we map the parameter region where this phenomenon is most prevalent. The strongest stretching and 

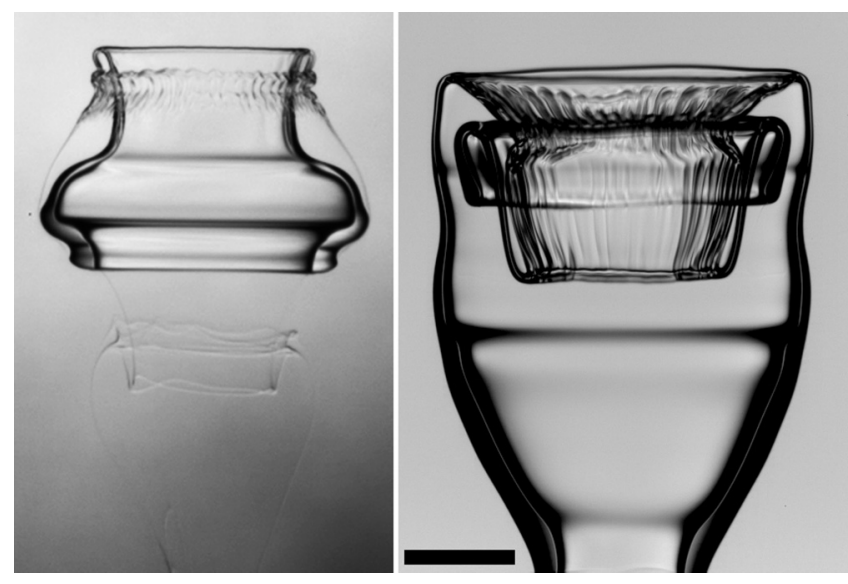

FIG. 30. Azimuthal buckling at the top side of the drop, for both high and low drop viscosities. The left panel is for the similar conditions as Fig. 3(c), with $v_{d}=500 \mathrm{cSt}, v_{p}=0.65 \mathrm{cSt}$ for $U=2.05 \mathrm{~m} / \mathrm{s}$, but shown at a much later time. (Right panel) Buckles occurring at the upper edge of the drop, during the later stages for conditions in Fig. 27, where $v_{d}=100 \mathrm{cSt}$. Scale bar is $1 \mathrm{~mm}$.

compression is induced around a vortex ring, which pulls in folds of the viscous sheet and winds them around its core. PIV allows us to track the origin and evolution of this vortex ring. The strong viscosity difference between drop and pool leads to a vortical boundary layer in the flow up along the bottom side of the drop liquid. This boundary layer separates at the top edge of the viscous bowl and rolls up to form the vortex ring, which subsequently advects into the bowl-shaped drop and starts entraining and winding up the viscous sheets, as is shown in Fig. 21. This vorticity-generation mechanism is qualitatively different from the vortex rings formed by the impact of a drop on an identical pool liquid, where it arises from the flow along the curved free air-liquid interface in the neck connecting the drop and pool [36,37]. Conceptually, if the drop impacts too fast for surface tension to pull the neck out radially, then the drop will contact with the pool at numerous places and no coherent vortex ring can be formed. This is known to occur at impact velocities above $\mathrm{We}_{c} \simeq 64$ [34]. Herein our strongest vortex rings are formed for We $\sim 400$, well above this critical value. Furthermore, the very viscous drop forms the top surface in the neck between drop and pool, which will eliminate the flow along the free surface inside the drop. It is this flow which provides the vorticity into the vortex ring, for the same-liquid drop
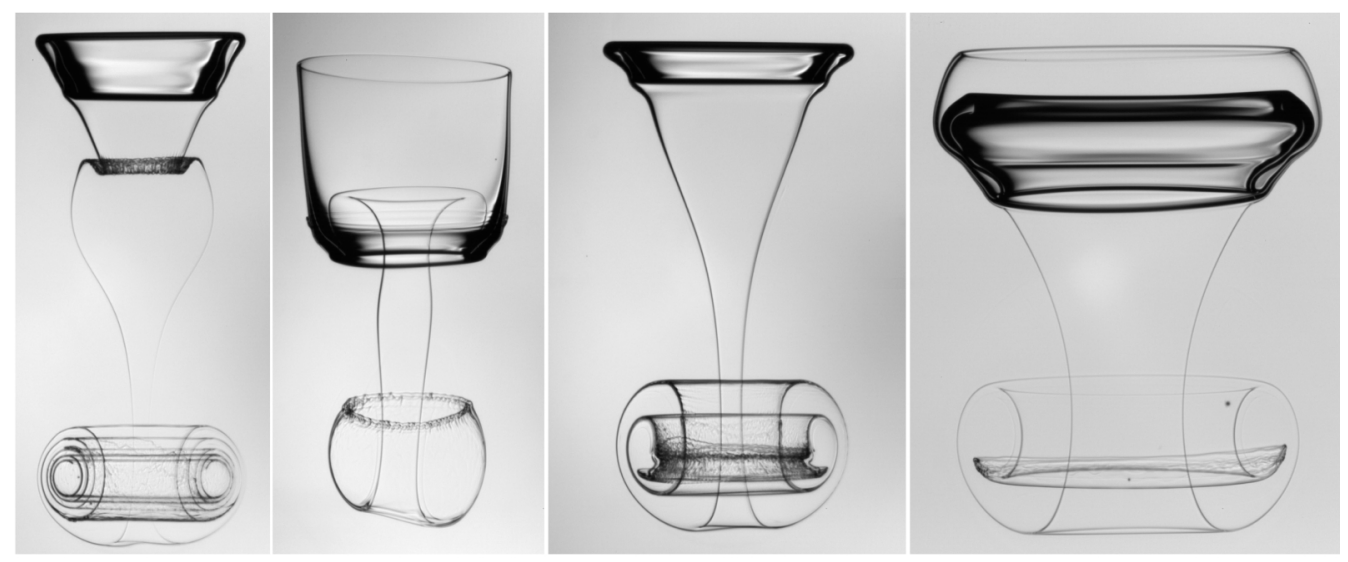

FIG. 31. Collage of additional shapes, showing the variety of formations observed. 
(a)

(b)

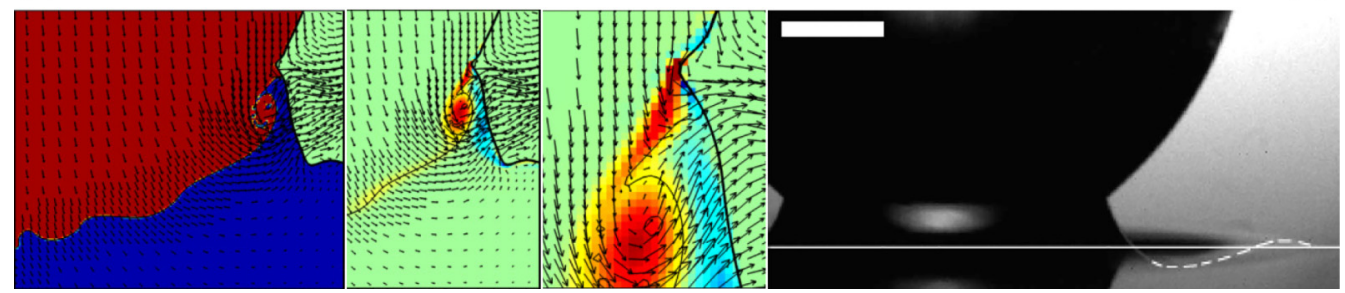

FIG. 32. (a) Vorticity production for a $D=5 \mathrm{~mm}$ water drop impacting on a water pool, at $U=1 \mathrm{~m} / \mathrm{s}$ (We $=68 ; \operatorname{Re}=5000)$, modified from Thoraval et al. [37]. The red color, in the second and third panels, indicates counterclockwise vorticity. The close-up shows the vorticity emerging from a sharp corner in the free surface within the drop liquid, marked red in the first panel. (b) Sharp corner in the neck between a pendent glycerin drop and a water pool, modified from Thoroddsen et al. [53]. Scale bar is $0.5 \mathrm{~mm}$.

and pool, as was shown in the simulations of Thoraval et al. [37] [see their Fig. 14, which is modified below in Fig. 32(a)]. It is known for low-viscosity pools that while the speed of the coalescing neck of a viscous drop can be greatly slowed by a triple line [52], it is not slowed down by the viscous drop when the two liquids are miscible [53]. However, the shape of the neck is likely to be greatly affected, as is shown, in Fig. 32(b), for the coalescence of static viscous drop with a low-viscosity pool. In addition, the flow along the free surface within the pool would produce vorticity of the opposite sign to that of the vortex ring. We therefore conclude that the flow around the bottom section of the impacting drop is not driven by surface tension but is more akin to stagnation-point flow moving radially outward along the near-immobile drop surface, thereby producing the vortical layer. Our PIV resolution cannot resolve this boundary layer at the very earliest times, but in Fig. 20, at $t=1.9 \mathrm{ms,}$ it is visible hugging the surface of the drop and is subsequently seen rolling up into the vortex ring.

One can wonder whether baroclinic generation of vorticity plays a role, when the drop liquid diffuses into the pool and is subject to the positive pressure gradient along the bottom of the drop. We do not expect this to be a significant effect, owing to the very large Schmidt number and modest density difference in this diffuse boundary layer. We expect the same observed mechanism to be at play even if the two liquids were of the same density.

It is of historical interest to note that our mechanism for generating the vortex ring is conceptually similar to that proposed by Thomson and Newall in their ground-breaking 1885 paper [1] for a drop impacting on an identical low-viscosity pool. They proposed erroneously that the impacting drop acted like a solid sphere, generating vorticity on its surface, with the solid drop subsequently dissolving into the pool, thereby leaving the vortex ring to propagate.

The video images allow us to measure the overall area of the drop liquid, which is relevant to stirring and mixing of different liquid masses. We find surprisingly rapid stretching where the total drop area can grow 40 -fold during the first $50 \mathrm{~ms}$. It is an intriguing possibility that additional folds are pulled in around the vortex ring, at later times, than are shown in Fig. 15, but these would be invisible, with our image magnifications, when they become submicron in thickness.

Even the simplest 2D model, i.e., that of a dye line wound up by a point vortex, reveals surprisingly rich behavior, with compression as well as stretching.

We have primarily focused on regular small-scale buckling of the viscous sheet of drop liquid. However, even the overall shape of the drop in Fig. 17 is in essence one fold of a buckle. The same is true of the first folding at the top of the bowl in Fig. 21.

Increasing the viscosity of the pool reduces the drop deformations and no buckling is observed. On the other hand, when the drop viscosity is reduced, the viscous sheets become even more convoluted and they buckle at much finer length scales.

Many of the transparent axisymmetric shapes resemble those of vases and are sometimes adorned with azimuthal undulations, some of which are shown in Figs. 30 and 31. We have only scratched the surface of the vast parameter space of this problem, where the two viscosities, two densities, 


\section{LI, BEILHARZ, AND THORODDSEN}

surface tension, drop diameter, and impact velocity can be varied. Future experiments are bound to reveal even more intriguing shapes and dynamics than have been presented herein.

\section{ACKNOWLEDGMENTS}

The work described herein was supported by King Abdullah University of Science and Technology (KAUST) research funding (URF/1/2621-01-01). Some of the videos in the Supplemental Material were submitted to the Gallery of Fluid Motions of the APS-DFD meeting held in Boston in November 2015. Li is grateful for the Thousand Young Talents Program of China, the National Natural Science Foundation of China (Grant No. 11621202), and the Fundamental Research Funds for the Central Universities (Grant No. WK2090050041). Beilharz was an intern at KAUST during the early phase of this work. We thank Joachim Delannoy for a helpful discussion on the modeling.

[1] J. Thomson and H. Newall, On the formation of vortex rings by drops falling into liquids and some allied phenomena, Proc. R. Soc. London 39, 417 (1885).

[2] G. Machu, W. Meile, L. C. Nitsche, and U. Schaflinger, Coalescence, torus formation and breakup of sedimenting drops: Experiments and computer simulations, J. Fluid Mech. 447, 299 (2001).

[3] M. Shimokawa, R. Mayumi, T. Nakamura, T. Takami, and H. Sakaguchi, Breakup and deformation of a droplet falling in a miscible solution, Phys. Rev. E 93, 062214 (2016).

[4] M. Ohta, Y. Akama, Y. Yoshida, and M. Sussman, Influence of the viscosity ratio on drop dynamics and breakup for a drop rising in an immiscible low-viscosity liquid, J. Fluid Mech. 752, 383 (2014).

[5] B. Metzger, M. Nicolas, and E. Guazzelli, Falling clouds of particles in viscous fluids, J. Fluid Mech. 580, 283 (2007).

[6] R. Pignatel, M. Nicolas, and É. Guazzelli, A falling cloud of particles at a small but finite Reynolds number, J. Fluid Mech. 671, 34 (2011).

[7] M. Landeau, R. Deguen, and P. Olson, Experiments on the fragmentation of a buoyant liquid volume in another liquid, J. Fluid Mech. 749, 478 (2014).

[8] M. Szymusiak, V. Sharma, L. C. Nitsche, and Y. Liu, Interaction of sedimenting drops in a miscible solution: Formation of heterogeneous toroidal-spiral particles, Soft Matter 8, 7556 (2012).

[9] T. Kong, J. Li, Z. Liu, Z. Zhou, P. H. Y. Ng, L. Wang, and H. C. Shum, Rapid mixing of viscous liquids by electrical coiling, Sci. Rep. 6, 19606 (2016).

[10] H. Lhuissier, C. Sun, A. Prosperetti, and D. Lohse, Drop Fragmentation at Impact Onto a Bath of an Immiscible Liquid, Phys. Rev. Lett. 110, 264503 (2013).

[11] T. Fujimatsu, H. Fujita, M. Hirota, and O. Okada, Interfacial deformation between an impacting water drop and a silicone-oil surface, J. Colloid Interface Sci. 264, 212 (2003).

[12] G. I. Taylor, Instability of jets, threads, and sheets of viscous fluid, in Proceedings of the Twelfth International Congress of Applied Mechanics, Stanford 1968 (Springer-Verlag, Berlin, 1969), p. 382.

[13] J. O. Cruickshank and B. R. Munson, Viscous fluid buckling of plane and axisymmetric jets, J. Fluid Mech. 113, 221 (1981).

[14] L. Mahadevan, W. S. Ryu, and A. D. T. Samuel, Fluid "rope-trick" investigated, Nature (London) 392, 140 (1998); correction 403, 502 (2000).

[15] M. Habibi, S. H. Hosseini, M. H. Khatami, and N. M. Ribe, Liquid supercoiling, Phys. Fluids 26, 024101 (2014).

[16] J. D. Buckmaster, A. Nachman, and L. Ting, The buckling and stretching of viscida, J. Fluid Mech. 69, 1 (1975).

[17] N. M. Ribe, Coiling of viscous jets, Proc. R. Soc. London, Ser. A 460, 3223 (2004).

[18] R. da Silveira, S. Chaieb, and L. Mahadevan, Rippling instability of a collapsing bubble, Science 287, 1468 (2000). 
[19] G. Debrégeas, P.-G. de Gennes, and F. Brochard-Wyart, The life and death of "bare" viscous bubble, Science 279, 1704 (1998).

[20] S. M. Suleiman and B. R. Munson, Viscous buckling of thin fluid layers, Phys. Fluids 24, 1 (1981).

[21] S. Battacharya, R. V. Craster, and M. R. Flynn, Buckling of a thin, viscous film in an axisymmetric geometry, Phys. Fluids 25, 043102 (2013).

[22] A. C. Slim, J. Teichman, and L. Mahadevan, Buckling of a thin-layer Couette flow, J. Fluid Mech. 694, 5 (2012).

[23] J. D. Paulsen, E. Hohlfeld, H. King, J. Huang, Z. Qiu, T. P. Russell, N. Menon, D. Vella, and B. Davidovitch, Curvature-induced stiffness and the spatial variation of wavelength in wrinkled sheets, Proc. Natl. Acad. Sci. USA 113, 1144 (2016).

[24] R. D. Schroll, M. Adda-Bedia, E. Cewrda, J. Huang, N. Menon, T. P. Russell, K. B. Toga, D. Vella, and B. Davidovitch, Capillary Deformations of Bendable Film, Phys. Rev. Lett. 111, 014301 (2013).

[25] T. Cubaud and T. G. Mason, Folding of Viscous Threads in Diverging Microchannels, Phys. Rev. Lett. 96, 114501 (2006).

[26] M. Le Merrer, D. Quéré, and C. Clanet, Buckling of Viscous Filaments of a Fluid Under Compression Stresses, Phys. Rev. Lett. 109, 064502 (2012).

[27] J. O. Marston, T. T. Truscott, N. B. Speirs, M. M. Mansoor, and S. T. Thoroddsen, Crown sealing and buckling instability during water entry of spheres, J. Fluid Mech. 794, 506 (2016).

[28] S. T. Thoroddsen, K. Takehara, T. G. Etoh, and C.-D. Ohl, Spray and microjets produced by focusing a laser pulse into a hemispherical drop, Phys. Fluids 21, 112101 (2009).

[29] T. W. Walker, A. N. Logia, and G. G. Fuller, Multiphase flow of miscible liquids: Jets and drops, Exp. Fluids 56, 106 (2015).

[30] D. An, A. Warning, K. G. Yancey, C.-T. Chang, V. R. Kern, A. K. Datta, P. H. Steen, D. Luo, and M. Ma, Mass production of shaped particles through vortex ring freezing, Nat. Commun. 7, 12401 (2016).

[31] K. Shariff and A. Leonard, Vortex rings, Annu. Rev. Fluid Mech. 24, 235 (1992).

[32] D. S. Chapman and P. R. Critchlow, J. Fluid Mech. 29, 177 (1967).

[33] F. Rodriguez and R. Mesler, J. Colloid Interface Sci. 121, 121 (1988).

[34] M. Hsiao, S. Lichter, and L. G. Quintero, The critical Weber number for vortex and jet formation for drops impinging on a liquid pool, Phys. Fluids 31, 3560 (1988).

[35] B. Peck and L. Sigurdson, The three-dimensional vortex structure of an impacting water drop, Phys. Fluids 6, 564 (1994).

[36] R. W. Cresswell and B. R. Morton, Drop-formed vortex rings: The generation of vorticity, Phys. Fluids 7 , 1363 (1995).

[37] M.-J. Thoraval, Y. Li, and S. T. Thoroddsen, Vortex-ring-induced large bubble entrainment during drop impact, Phys. Rev. E 93, 033128 (2016).

[38] G. Agbaglah, M.-J. Thoraval, S. T. Thoroddsen, L. V. Zhang, K. Fezzaa, and R. D. Deegan, Drop impact into a deep pool: Vortex shedding and jet formation, J. Fluid Mech. 764, R1 (2015).

[39] J. Lee, S. J. Park, J. H. Lee, B. M. Weon, K. Fezzaa, and J. H. Je, Origin and dynamics of vortex rings in drop splashing, Nat. Commun. 6, 8187 (2015).

[40] D. Beilharz, A. Guyon, E. Q. Li, M.-J. Thoraval, and S. T. Thoroddsen, Antibubbles and fine cylindrical sheets of air, J. Fluid Mech. 779, 87 (2015).

[41] S. T. Thoroddsen, T. G. Etoh, and K. Takehara, Air entrapment under an impacting drop, J. Fluid Mech. 478, 125 (2003).

[42] M. J. McPhail, A. A. Fontaine, M. H. Krane, L. Goss, and J. Crafton, Correcting for color crosstalk and chromatic aberration in multicolor particle shadow velocimetry, Meas. Sci. Technol. 26, 025302 (2015).

[43] See Supplemental Material at http://link.aps.org/supplemental/10.1103/PhysRevFluids.2.073602 for videos.

[44] S. T. Thoroddsen, M.-J. Thoraval, K. Takehara, and T. G. Etoh, Micro-bubble morphologies following drop impacts onto a pool surface, J. Fluid Mech. 708, 469 (2012).

[45] J. O. Marston and S. T. Thoroddsen, Apex jets from impacting drops, J. Fluid Mech. 614, 293 (2008).

[46] M. H. W. Hendrix, W. Bouwhuis, D. van der Meer, D. Lohse, and J. H. Snoeijer, Universal mechanism for air entrainment during liquid impact, J. Fluid Mech. 789, 708 (2016). 


\section{LI, BEILHARZ, AND THORODDSEN}

[47] F. Durst, Penetration length and diameter development of vortex rings generated by impacting water drops, Exp. Fluids 21, 110117 (1996).

[48] N. Rashidnia, R. Balasubramaniam, J. Kuang, P. Petitjeans, and T. Maxworthy, Measurement of the diffusion coefficient of miscible fluids using both interferometry and Wiener's method, Int. J. Thermophys. 22, 547 (2001).

[49] G. K. Batchelor, An Introduction to Fluid Dynamics (Cambridge University Press, Cambridge, UK, 1967).

[50] M. Brøns, M. C. Thompson, T. Leweke, and K. Hourigan, Vorticity generation and conservation for two-dimensional interfaces and boundaries, J. Fluid Mech. 758, 63 (2014).

[51] M.-J. Thoraval, K. Takehara, T. G. Etoh, S. Popinet, P. Ray, C. Josserand, S. Zaleski, and S. T. Thoroddsen, Von Kármán Vortex Street within an Impacting Drop, Phys. Rev. Lett. 108, 264506 (2012).

[52] E. Q. Li, S. Al-Otaibi, I. U. Vakarelski, and S. T. Thoroddsen, Satellite formation during bubble transition through an interface between immiscible liquids, J. Fluid Mech. 744, R1 (2014).

[53] S. T. Thoroddsen, B. Qian, T. G. Etoh, and K. Takehara, The initial coalescence of miscible drops, Phys. Fluids 19, 072110 (2007). 